\title{
Oxygen-to-Oxygen Silyl Migration of Alpha-Siloxy Sulfoxides and Oxidation-triggered Allicin Formation
}

\author{
Shane S. Kelly, ${ }^{a, b}$ Tun-Li Shen, ${ }^{a}$ Ming Xian ${ }^{a *}$ \\ aDepartment of Chemistry, Brown University, Providence, RI 02912, USA

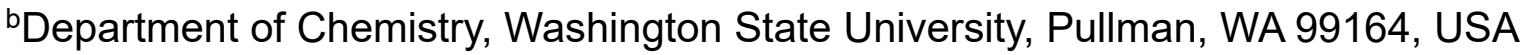

Materials and Methods. All commercially obtained reagents were used as received unless otherwise stated. All reactions were performed at room temperature (approximately $21^{\circ} \mathrm{C}$ ) unless stated otherwise. All flash chromatography was performed using 230-400 mesh silica gel. All ${ }^{1} \mathrm{H}$ NMR spectra were obtained from Varian $(600 \mathrm{MHz}$ and $400 \mathrm{MHz})$ or Bruker $(600 \mathrm{MHz}$ and $400 \mathrm{MHz}$ ) spectrometers. All ${ }^{1} \mathrm{H}$ NMR spectra are reported relative to the residual solvent signal ( $\delta 7.26$ for $\left.\mathrm{CDCl}_{3}\right)$. All ${ }^{13} \mathrm{C}$ NMR spectra were obtained from Varian $(151 \mathrm{MHz}$ and 101 $\mathrm{MHz}$ ) or Bruker (151 MHz and $101 \mathrm{MHz}$ ) spectrometers. All ${ }^{13} \mathrm{C}$ NMR spectra are reported relative to the residual solvent signal $\left(\delta 77.16\right.$ for $\left.\mathrm{CDCl}_{3}\right)$ High resolution mass spectra were obtained using an Agilent 6530 (Q-TOF) LC-MS in ${ }^{+}$ESI mode. El spectra were obtained using an Agilent 5977A GC-MS. Low resolution ESI (+/-) mass spectra were obtained using either Thermo LXQ linear ion trap LC-MS or Thermo LCQ Deca XP Max LC-MS. HPLC was performed using an Agilent 1260 Infinite LC system with diode array detector $(\lambda=254 \mathrm{~nm}$ and $214 \mathrm{~nm})$. 


\section{Experimental Procedures:}

\section{General Procedure 1 for Synthesis of alpha-siloxysulfides}

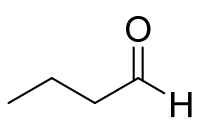

SI-1

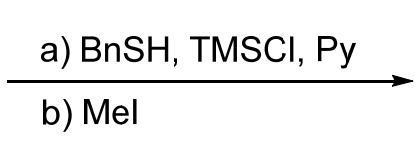

b) Mel

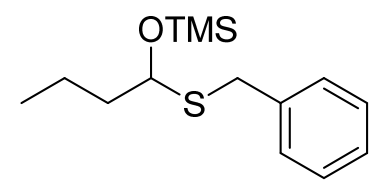

$6 a$

Compound 6a. A $10 \mathrm{~mL}$ flame-dried round-bottom flask was charged with dry pyridine $(4.0$ $\mathrm{mL})$, followed by addition of butyraldehyde $(9.6 \mathrm{mmol}, 0.87 \mathrm{~mL}, 1.2 \mathrm{eq})$ before cooling to $0^{\circ} \mathrm{C}$. After addition of TMSCl $(11.2 \mathrm{mmol}, 1.42 \mathrm{~mL}, 1.4 \mathrm{eq})$, benzyl mercaptan $(8.0 \mathrm{mmol}, 0.94 \mathrm{~mL}$, 1.0 eq) was diluted with pyridine $(1 \mathrm{~mL})$ and added dropwise. The reaction was stirred for 30 minutes at $0^{\circ} \mathrm{C}$, then for 18 hours at room temperature. Mel $(16.0 \mathrm{mmol}, 0.99 \mathrm{~mL}, 2.0 \mathrm{eq})$ was added to the flask and stirred for 2 hours. Two yellow liquid phases appeared, and the bottom phase was discarded. (Note: The reaction may also present itself as a yellow liquid with abundant yellow precipitate, in which case the precipitate was filtered and discarded. Mel was added to quench residual thiol and facilitate purification). The liquid was concentrated in vacuo and crude oil purified by flash chromatography (15\% ethyl acetate/hexanes), yielding $6 \mathbf{a}$ as a clear/colorless oil. (1.31 g, $4.9 \mathrm{mmol}, 61 \%) .{ }^{1} \mathrm{H}$ NMR $\left(400 \mathrm{MHz}, \mathrm{CDCl}_{3}\right) \delta 7.37-7.18(\mathrm{~m}, 5 \mathrm{H})$, $4.81(\mathrm{t}, J=6.4 \mathrm{~Hz}, 1 \mathrm{H}), 3.81(\mathrm{~s}, 2 \mathrm{H}), 1.86-1.64(\mathrm{~m}, 2 \mathrm{H}), 1.40(\mathrm{tdd}, J=14.1,7.7,1.1 \mathrm{~Hz}, 2 \mathrm{H})$, 0.88 (t, $J=7.4 \mathrm{~Hz}, 3 \mathrm{H}), 0.17$ (s, 9H); ${ }^{13} \mathrm{C}$ NMR $\left(101 \mathrm{MHz}, \mathrm{CDCl}_{3}\right) \delta$ 138.7, 129.1, 128.6, 127.0, 78.6, 41.0, 33.3, 19.5, 13.8, 0.7; MS-El m/z: $\left[\mathrm{M}-\mathrm{CH}_{3}\right]^{+} \mathrm{C}_{13} \mathrm{H}_{21} \mathrm{OSSi}$ calcd 253.1, found 253; [MTMS $]^{+} \mathrm{C}_{11} \mathrm{H}_{15} \mathrm{OS}$ calcd 195.1, found 195 ; [M] ${ }^{+} \mathrm{C}_{14} \mathrm{H}_{24} \mathrm{OSSi}$ calcd 268.1, found 268. Note: for some reason, these $\alpha$-siloxysulfide compounds (6a-d and 17a-c) appear to be unstable under mass analysis. We have tried different ionization methods ( $E S I, E I, A P C l, M A L D I)$ to obtain their parent MS and HRMS. We were only able to obtain a few parent MS using El. For each 
and derivatization confirmed their structural identity.

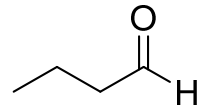

SI-1

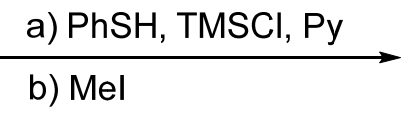

b) Mel

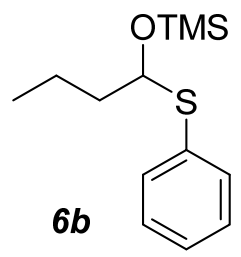

Compound 6b. Clear/colorless oil, $1.95 \mathrm{~g}, 7.7 \mathrm{mmol}, 96 \% ;{ }^{1} \mathrm{H}$ NMR (600 MHz, CDCl $) \delta 7.53-$ $7.48(\mathrm{~m}, 2 \mathrm{H}), 7.33-7.26(\mathrm{~m}, 3 \mathrm{H}), 5.09(\mathrm{t}, J=6.3 \mathrm{~Hz}, 1 \mathrm{H}), 1.75\left(\mathrm{~m}^{`}, 2 \mathrm{H}\right), 1.53-1.38(\mathrm{~m}, 2 \mathrm{H})$, $0.91(\mathrm{t}, J=7.4 \mathrm{~Hz}, 3 \mathrm{H}), 0.04(\mathrm{~s}, 9 \mathrm{H}) ;{ }^{13} \mathrm{C} \mathrm{NMR}\left(151 \mathrm{MHz}, \mathrm{CDCl}_{3}\right) \delta$ 134.0, 134.0, 128.8, 127.7, 82.6, 41.0, 19.6, 13.8, 0.3; MS-El m/z: [M] ${ }^{+} \mathrm{C}_{13} \mathrm{H}_{22} \mathrm{OSSi}$ calcd 254.1, found 254; [M-CH3] ${ }^{+}$ $\mathrm{C}_{12} \mathrm{H}_{19} \mathrm{OSSi}$ calcd 239.1, found 239; [M-TMS] ${ }^{+} \mathrm{C}_{10} \mathrm{H}_{13} \mathrm{OS}$ calcd 181.1, found 181.<smiles>CCCC=O</smiles>

SI-1

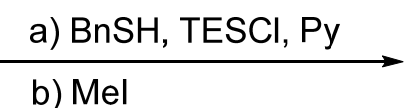

b) Mel

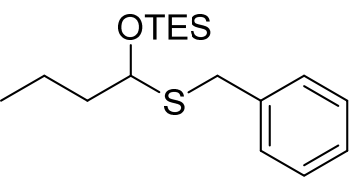

$6 c$

Compound 6c. Clear/colorless oil, 2.0 g, 6.4 mmol, 80\%; ${ }^{1} \mathrm{H}$ NMR (600 MHz, CDCl $)$ $\delta 7.34$ (d, $J=7.3 \mathrm{~Hz}, 2 \mathrm{H}), 7.30(\mathrm{t}, J=7.6 \mathrm{~Hz}, 2 \mathrm{H}), 7.23(\mathrm{t}, J=7.2 \mathrm{~Hz}, 1 \mathrm{H}), 4.82(\mathrm{t}, J=6.4 \mathrm{~Hz}, 1 \mathrm{H}), 3.83$ (s, 2H), 1.77 (dtd, $J=40.0,13.9,7.6 \mathrm{~Hz}, 2 \mathrm{H}), 1.42(\mathrm{~h}, J=7.5 \mathrm{~Hz}, 2 \mathrm{H}), 0.99(\mathrm{t}, J=8.0 \mathrm{~Hz}, 9 \mathrm{H})$, $0.89(\mathrm{t}, J=7.4 \mathrm{~Hz}, 3 \mathrm{H}), 0.67(\mathrm{q}, J=7.9 \mathrm{~Hz}, 6 \mathrm{H}) ;{ }^{13} \mathrm{C}$ NMR $\left(151 \mathrm{MHz}, \mathrm{CDCl}_{3}\right) \delta$ 138.7, 129.1, 128.5, 126.9, 78.6, 41.3, 32.9, 19.5, 13.9, 7.0, 5.2; MS-El m/z: [M] ${ }^{+} \mathrm{C}_{17} \mathrm{H}_{30} \mathrm{OSSi}$ calcd 310.2, found 310; [M-TES] ${ }^{+} \mathrm{C}_{11} \mathrm{H}_{15} \mathrm{OS}$ calcd 195.1, found 195.

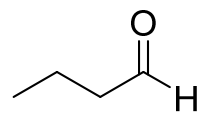

SI-1

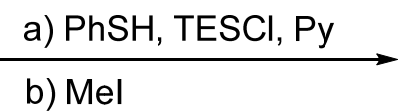

b) Mel<smiles></smiles> 
Compound 6d. Clear/colorless oil, $1.54 \mathrm{~g}, 5.2 \mathrm{mmol}, 66 \%$; ${ }^{1} \mathrm{H}$ NMR $\left(400 \mathrm{MHz}, \mathrm{CDCl}_{3}\right) \delta 7.54$ $-7.45(\mathrm{~m}, 2 \mathrm{H}), 7.35-7.21(\mathrm{~m}, 3 \mathrm{H}), 5.10(\mathrm{t}, J=6.3 \mathrm{~Hz}, 1 \mathrm{H}), 1.83-1.66(\mathrm{~m}, 2 \mathrm{H}), 1.54-1.39$ (m, 2H), 0.99-0.85 (m, 12H), $0.58(\mathrm{q}, J=7.6 \mathrm{~Hz}, 6 \mathrm{H}) ;{ }^{13} \mathrm{C}$ NMR $\left(101 \mathrm{MHz}, \mathrm{CDCl}_{3}\right) \delta$ 134.0, 133.7, 128.8, 127.5, 82.4, 41.1, 19.6, 13.9, 6.9, 5.0; MS-El m/z: [M-TES] ${ }^{+} \mathrm{C}_{10} \mathrm{H}_{13} \mathrm{OS}$ calcd 181.1, found 181.

\section{General Procedure 2 for Synthesis of alpha-siloxysulfides}

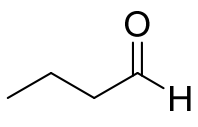

SI-1

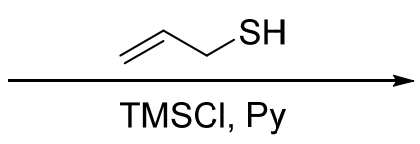

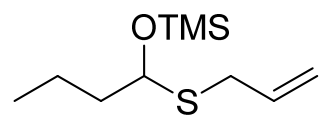

$17 a$

Compound 17a. A $10 \mathrm{~mL}$ flame-dried round-bottom flask was charged with dry pyridine (4.0 $\mathrm{mL})$, followed by addition of butyraldehyde $(9.6 \mathrm{mmol}, 0.87 \mathrm{~mL}, 1.2 \mathrm{eq})$ before cooling to $0^{\circ} \mathrm{C}$. After addition of TMSCI (11.2 mmol, $1.41 \mathrm{~mL}, 1.4 \mathrm{eq})$, allyl mercaptan ( $\geq 70 \%$ pure, $8.0 \mathrm{mmol}$, $0.94 \mathrm{~mL}, 1.0 \mathrm{eq})$ was diluted with pyridine $(1 \mathrm{~mL})$ and added dropwise. The reaction was stirred for 30 minutes at $0^{\circ} \mathrm{C}$, then for 18 hours at room temperature. The white precipitate was filtered and discarded. The liquid was concentrated in vacuo and crude oil purified by flash chromatography ( $10 \%$ dichloromethane/hexanes), yielding $17 \mathrm{a}$ as a somewhat volatile clear/colorless oil. (0.70 g, $3.2 \mathrm{mmol}, 40 \%) .{ }^{1} \mathrm{H}$ NMR (400 MHz, $\left.\mathrm{CDCl}_{3}\right) \delta 5.85$ (ddt, $J=16.9$, 10.0, $6.7 \mathrm{~Hz}, 1 \mathrm{H}), 5.16(\mathrm{dq}, J=17.0,1.4 \mathrm{~Hz}, 1 \mathrm{H}), 5.07(\mathrm{dd}, J=10.0,1.4 \mathrm{~Hz}, 1 \mathrm{H}), 4.84(\mathrm{t}, J=$ $6.3 \mathrm{~Hz}, 1 \mathrm{H}), 3.31-3.16(\mathrm{~m}, 2 \mathrm{H}), 1.84-1.64(\mathrm{~m}, 2 \mathrm{H}), 1.48-1.35(\mathrm{~m}, 2 \mathrm{H}), 0.91(\mathrm{t}, J=7.4 \mathrm{~Hz}$, 3H), 0.17 (s, 9H); ${ }^{13} \mathrm{C}$ NMR (101 MHz, $\left.\mathrm{CDCl}_{3}\right) \delta$ 135.1, 116.8, 78.2, 41.1, 32.0, 19.5, 13.9, 0.7; MS-El m/z: $\left[\mathrm{M}-\mathrm{CH}_{3}\right]^{+} \mathrm{C}_{9} \mathrm{H}_{19} \mathrm{OSSi}$ calcd 203.1, found 203; [M-TMS] ${ }^{+} \mathrm{C}_{7} \mathrm{H}_{13} \mathrm{OS}$ calcd 145.1, found 145 . 


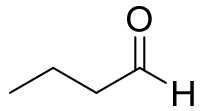

SI-1

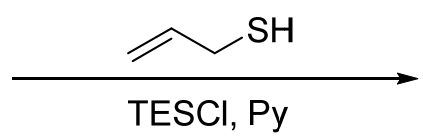

TESCl, Py

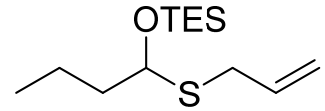

$17 b$

Compound 17b. Clear/colorless oil, $1.13 \mathrm{~g}, 4.3 \mathrm{mmol}, 54 \% ;{ }^{1} \mathrm{H}$ NMR $\left(600 \mathrm{MHz}, \mathrm{CDCl}_{3}\right) \delta 5.92-$ $5.82(\mathrm{~m}, 1 \mathrm{H}), 5.16(\mathrm{~d}, J=17,1 \mathrm{H}), 5.06(\mathrm{~d}, \mathrm{~J}=11,1 \mathrm{H}), 4.84(\mathrm{t}, J=6.3 \mathrm{~Hz}, 1 \mathrm{H}), 3.32-3.19(\mathrm{~m}$, $2 \mathrm{H}), 1.85-1.68(\mathrm{~m}, 2 \mathrm{H}), 1.49-1.38(\mathrm{~m}, 2 \mathrm{H}), 0.99(\mathrm{t}, J=8.0 \mathrm{~Hz}, 9 \mathrm{H}), 0.92(\mathrm{t}, J=7.4 \mathrm{~Hz}, 3 \mathrm{H})$, $0.67(\mathrm{q}, J=8.0 \mathrm{~Hz}, 6 \mathrm{H}) ;{ }^{13} \mathrm{C} \mathrm{NMR}\left(151 \mathrm{MHz}, \mathrm{CDCl}_{3}\right) \delta 135.3,116.6,78.4,41.5,31.7,19.5$, 13.9, 7.0, 5.3; MS-El m/z: [M-TES] ${ }^{+} \mathrm{C}_{7} \mathrm{H}_{13} \mathrm{OS}$ calcd 145.1, found 145.

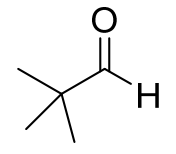

SI-2

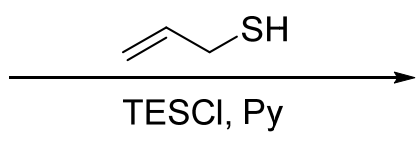

TESCl, Py

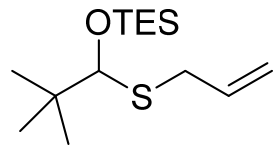

$17 c$

Compound 17c. Clear/colorless oil, $1.5 \mathrm{~g}, 5.4 \mathrm{mmol}, 67 \%$; ${ }^{1} \mathrm{H}$ NMR $\left(400 \mathrm{MHz}, \mathrm{CDCl}_{3}\right) \delta 5.93$ $5.75(\mathrm{~m}, 1 \mathrm{H}), 5.13(\mathrm{dq}, J=17.0,1.4 \mathrm{~Hz}, 1 \mathrm{H}), 5.10-5.04(\mathrm{~m}, 1 \mathrm{H}), 4.45(\mathrm{~s}, 1 \mathrm{H}), 3.25(\mathrm{dt}, J=$ 7.1, $1.1 \mathrm{~Hz}, 2 \mathrm{H}), 1.06-0.92(\mathrm{~m}, 18 \mathrm{H}), 0.76-0.59(\mathrm{~m}, 6 \mathrm{H}) ;{ }^{13} \mathrm{C} \mathrm{NMR}\left(101 \mathrm{MHz}, \mathrm{CDCl}_{3}\right) \delta$ 135.3, 116.8, 89.2, 39.1, 35.2, 26.4, 7.1, 5.4; MS-El m/z: [M-TES] ${ }^{+} \mathrm{C}_{8} \mathrm{H}_{15} \mathrm{OS}$ calcd 159.1; found 159; [M-CH$]_{3}^{+} \mathrm{C}_{13} \mathrm{H}_{27} \mathrm{OSSi}$ calcd 259.1, found 259 .

\section{Stability studies of alpha-siloxysulfides}

Each alpha-siloxysulfide substrate (6a-d) was dissolved in 1:1 of THF/buffers (pH 5 9) and stored at rt. Their degradation was monitored by TLC. After 24 hours the materials were extracted by DCM and analyzed by NMR. No significant decomposition was noted as all materials were recovered $>95 \%$.

\section{Identification of silyl migration reaction products}




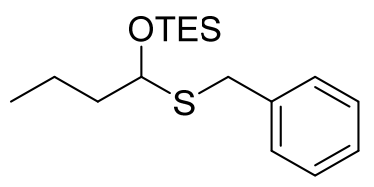

$6 c$

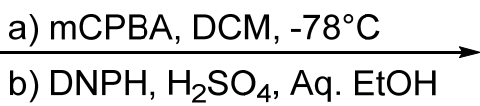

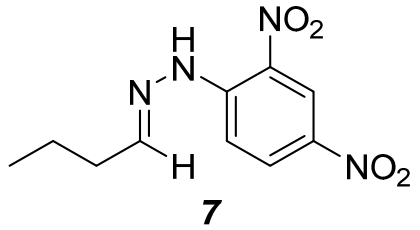

Compound 7. A $100 \mathrm{~mL}$ round-bottom flask was charged with $38 \mathrm{~mL}$ dichloromethane and $6 \mathrm{c}$ $(1.20 \mathrm{mmol}, 0.373 \mathrm{~g}, 1.0 \mathrm{eq})$ prior to evacuation with argon and cooling to $-78^{\circ} \mathrm{C} . \mathrm{mCPBA}$ ( $70 \%$ pure, $1.08 \mathrm{mmol}, 0.266 \mathrm{~g}, 0.9 \mathrm{eq})$ dissolved in $10 \mathrm{~mL}$ dichloromethane was then added by syringe pump over 30 minutes and reaction stirred for 3 hours at $-78^{\circ} \mathrm{C}$. The reaction was warmed to room temperature and stirred for 1 hour with a solution of 2,4dinitrophenylhydrazine (1.44 mmol, $0.36 \mathrm{~g}, 1.2 \mathrm{eq})$ dissolved in $\mathrm{H}_{2} \mathrm{SO}_{4}(1.5 \mathrm{~mL}), \mathrm{H}_{2} \mathrm{O}(2.5 \mathrm{~mL})$, and $95 \% \mathrm{EtOH}(7.5 \mathrm{~mL})$. It was then diluted with dichloromethane $(20 \mathrm{~mL})$ and organic phase washed (1x, $\left.15 \mathrm{~mL}, 1 \mathrm{M} \mathrm{H}_{2} \mathrm{SO}_{4}\right)$ and $\left(3 \mathrm{x}, 15 \mathrm{~mL}\right.$, sat. $\left.\mathrm{NaHCO}_{3}\right)$ and $(1 \mathrm{x}, 10 \mathrm{~mL}$, brine) and dried with $\mathrm{MgSO}_{4}$. After filtration and concentration in vacuo, the crude material was purified by flash chromatography (10\% ethyl acetate/hexanes) to yield 7 as an orange solid $(1.1 \mathrm{mmol}$, $0.27 \mathrm{~g}, 89 \%) .{ }^{1} \mathrm{H}$ NMR (400 MHz, $\left.\mathrm{CDCl}_{3}\right) \delta 11.02(\mathrm{br} \mathrm{s}, 1 \mathrm{H}), 9.13(\mathrm{~d}, J=2.6 \mathrm{~Hz}, 1 \mathrm{H}), 8.30$ (dd, $J=9.6,2.6 \mathrm{~Hz}, 1 \mathrm{H}), 7.93(\mathrm{~d}, J=9.6 \mathrm{~Hz}, 1 \mathrm{H}), 7.54(\mathrm{t}, J=5.4 \mathrm{~Hz}, 1 \mathrm{H}), 2.42(\mathrm{td}, J=7.4,5.4 \mathrm{~Hz}$, 2H), $1.71-1.61(\mathrm{~m}, 2 \mathrm{H}), 1.03(\mathrm{t}, J=7.4 \mathrm{~Hz}, 3 \mathrm{H}){ }^{1}$

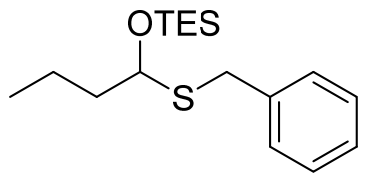

$6 c$

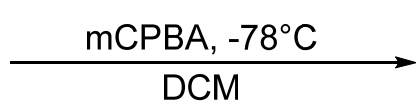

DCN

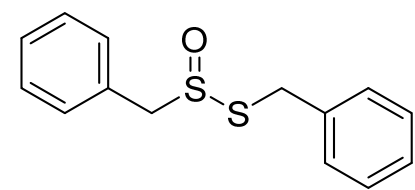

13

Compound 13. A $100 \mathrm{~mL}$ round-bottom flask was charged with $38 \mathrm{~mL}$ dichloromethane and $6 \mathrm{c}$ ( $1.20 \mathrm{mmol}, 0.373 \mathrm{~g}, 1.0 \mathrm{eq})$ prior to cooling to $-78^{\circ} \mathrm{C}$. mCPBA ( $70 \%$ pure, $1.08 \mathrm{mmol}, 0.266$ $\mathrm{g}, 0.9 \mathrm{eq}$ ) dissolved in $10 \mathrm{~mL}$ dichloromethane was then added by syringe pump over 30 minutes. The reaction stirred for 3 hours at $-78^{\circ} \mathrm{C}$ before warming and addition of saturated $\mathrm{Na}_{2} \mathrm{~S}_{2} \mathrm{O}_{3}(10 \mathrm{~mL})$. The organic phase was washed $\left(3 \mathrm{x}, 15 \mathrm{~mL}\right.$, sat. $\left.\mathrm{NaHCO}_{3}\right)$ and $(1 \mathrm{x}, 10 \mathrm{~mL}$, 
brine), dried with $\mathrm{MgSO}_{4}$, filtered, and concentrated in vacuo. The crude material was purified by flash chromatography ( $100 \%$ dichloromethane) to yield 13 as a white solid $(0.11 \mathrm{~g}, 0.4$ mmol, 80\%). ${ }^{1} \mathrm{H}$ NMR (600 MHz, $\left.\mathrm{CDCl}_{3}\right) \delta 7.40-7.24(\mathrm{~m}, 10 \mathrm{H}), 4.36-4.24(\mathrm{~m}, 4 \mathrm{H}) ;{ }^{13} \mathrm{C} \mathrm{NMR}$ $\left(151 \mathrm{MHz}, \mathrm{CDCl}_{3}\right) \delta 136.8,130.5,130.1,129.3,129.0,128.9,127.9,62.4,36.3 ; \mathrm{HRMS}\left({ }^{+} \mathrm{ESI}\right.$ in $\mathrm{ACN}$ ) calcd for $\mathrm{C}_{14} \mathrm{H}_{15} \mathrm{OS}_{2}{ }^{+}[\mathrm{M}+\mathrm{H}]^{+} 263.0559$, found $263.0572 .^{2}$

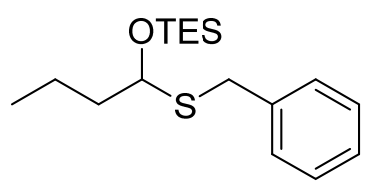

$6 c$

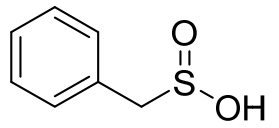

10

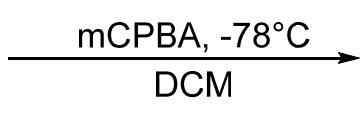

13

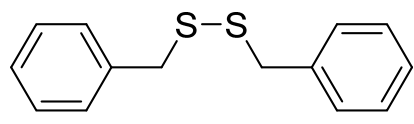

9

Compounds $9 \& 10$. A $25 \mathrm{~mL}$ two-neck round-bottom flask was sealed and evacuated with argon. It was charged with dichloromethane $(6 \mathrm{~mL})$ and $6 \mathrm{c}(0.2 \mathrm{mmol}, 0.054 \mathrm{~g}, 1.0 \mathrm{eq})$ prior to cooling to $-78^{\circ} \mathrm{C}$. mCPBA ( 70\% pure, $\left.0.18 \mathrm{mmol}, 0.044 \mathrm{~g}, 0.9 \mathrm{eq}\right)$ dissolved in $2 \mathrm{~mL}$ dichloromethane was added by syringe pump over 30 minutes, followed by stirring for 3 hours at $-78^{\circ} \mathrm{C}$. The reaction was warmed to room temperature for 48 hours prior to El-GCMS and -ESIMS analysis. Compound 10 -ESI-MS $[\mathrm{M}-\mathrm{H}]^{-} \mathrm{C}_{7} \mathrm{H}_{7} \mathrm{O}_{2} \mathrm{~S}$ calcd 155.0, found 155. Compound 9 ElGCMS $m / z[M]^{+} \mathrm{C}_{14} \mathrm{H}_{14} \mathrm{~S}_{2}$ calcd 246.0, found 246 (NIST spectral match).

\section{Crossover studies}

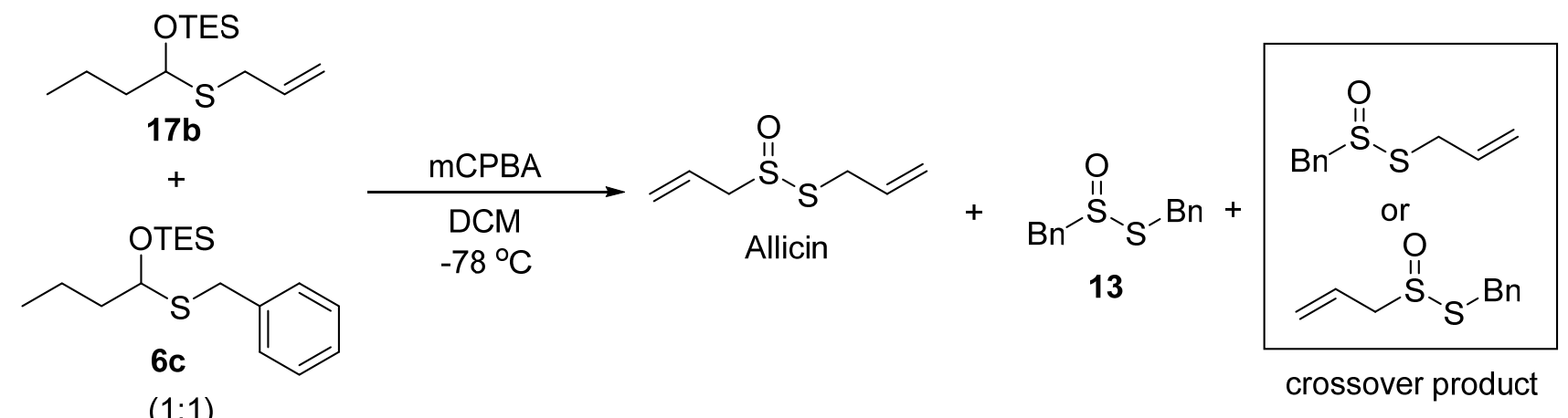


A $250 \mathrm{~mL}$ round-bottom flask was charged with $60 \mathrm{~mL} \mathrm{DCM}, 17 \mathrm{~b}(1.00 \mathrm{mmol}, 0.261 \mathrm{~g}, 1.0 \mathrm{eq})$, and $6 \mathrm{c}(1.00 \mathrm{mmol}, 0.311 \mathrm{~g}, 1.0 \mathrm{eq})$ prior to cooling to $-78^{\circ} \mathrm{C}$. mCPBA $(\sim 70 \%$ pure, $1.80 \mathrm{mmol}$, $0.444 \mathrm{~g}, 1.8 \mathrm{eq}$ ) dissolved in $20 \mathrm{~mL}$ dichloromethane was then added by syringe pump over 30 minutes. The reaction stirred for 3 hours at $-78^{\circ} \mathrm{C}$ before warming and addition of saturated $\mathrm{Na}_{2} \mathrm{~S}_{2} \mathrm{O}_{3}(10 \mathrm{~mL})$. The organic phase was washed $\left(3 \mathrm{x}, 15 \mathrm{~mL}\right.$, sat. $\left.\mathrm{NaHCO}_{3}\right)$ and $(1 \mathrm{x}, 10 \mathrm{~mL}$, brine), dried with $\mathrm{MgSO}_{4}$, filtered, and concentrated in vacuo. The crude material was analyzed by HPLC and HRMS. As shown below, in addition to the two self-condensation products (allicin and 13), an additional product peak (RT at $4.543 \mathrm{~min}$ ) was observed. HRMS demonstrated that it was the crossover product.

HPLC was done using a Supelcosil ${ }^{\mathrm{TM}} \mathrm{LC}-18$ column $(50 \times 4.6 \mathrm{~mm}, 5 \mu \mathrm{m}), \mathrm{ACN} / \mathrm{H}_{2} \mathrm{O}=5 / 95$ to 95/5 gradient, flow rate $=2.0 \mathrm{~mL} / \mathrm{min}, \mathrm{I}=214 \mathrm{~nm}$.

HPLC Trace:

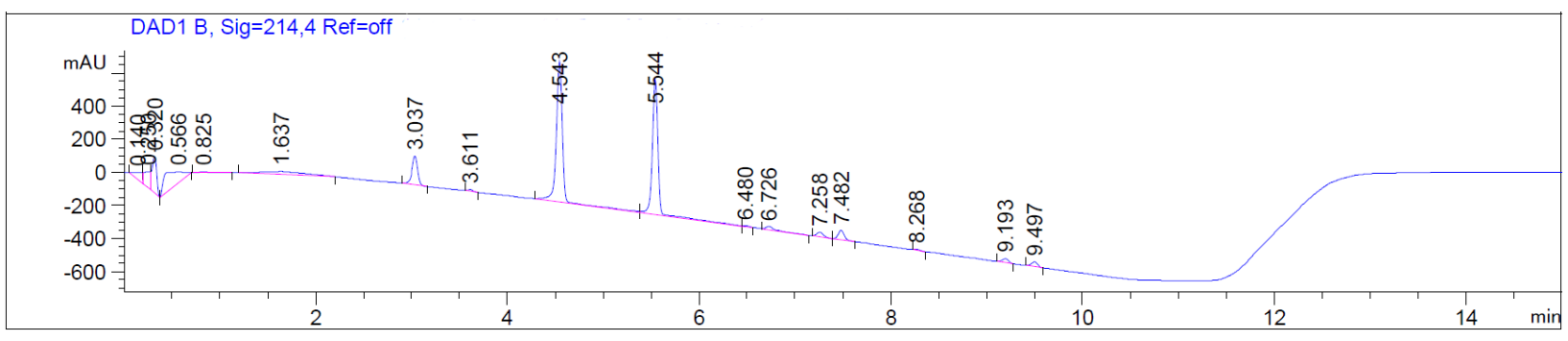

RT 3.037 min, Allicin. HRMS ( ${ }^{+} \mathrm{ESI}$ ) calcd for $\mathrm{C}_{6} \mathrm{H}_{11} \mathrm{OSS}_{2}{ }^{+}[\mathrm{M}+\mathrm{H}]^{+}$163.0246, found 3163.0251.

RT $4.543 \mathrm{~min}$, the crossover thiosulfinates. HRMS ( ${ }^{+} \mathrm{ESI}$ ) calcd for $\mathrm{C}_{10} \mathrm{H}_{13} \mathrm{OS}_{2}{ }^{+}[\mathrm{M}+\mathrm{H}]^{+}$ 213.0402, found 213.0413 .

RT 5.544 min, dibenzyl thiosulfinate 13. HRMS ( $\left.{ }^{+} E S I\right)$ calcd for $\mathrm{C}_{14} \mathrm{H}_{15} \mathrm{OS}_{2}{ }^{+}[\mathrm{M}+\mathrm{H}]^{+} 263.0559$, found 263.0571 .

\section{Synthesis of mechanistically relevant analogs}




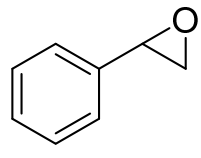

SI-3

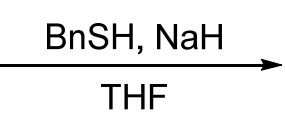

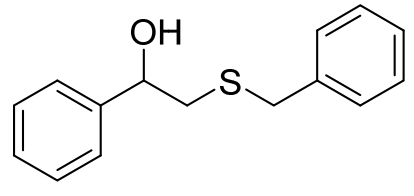

SI-4

Compound SI-4. A $10 \mathrm{~mL}$ two-neck flame-dried round-bottom flask was charged with THF (5 $\mathrm{mL}$ ) and benzyl mercaptan (6.2 mmol, $0.77 \mathrm{~g}, 2.5 \mathrm{eq})$. Sodium hydride (excess) was added and stirred for 20 minutes prior to dropwise addition of SI-3 $(2.5 \mathrm{mmol}, 0.30 \mathrm{~g}, 1.0 \mathrm{eq})$. After stirring for 18 hours, saturated ammonium chloride $(5 \mathrm{~mL})$ was added, and the resulting mixture was diluted with ethyl acetate $(60 \mathrm{~mL})$, organic phase washed $\left(3 \mathrm{x}, 10 \mathrm{~mL}\right.$, sat. $\left.\mathrm{NaHCO}_{3}\right)$ and $\left(1 \mathrm{x}, 10 \mathrm{~mL}\right.$, brine), then dried with $\mathrm{MgSO}_{4}$. Filtration and concentration in vacuo gave the crude material, which was further purified by flash chromatography $(10 \%$ ethyl acetate/hexanes) to yield SI-4 as a white solid $(1.9 \mathrm{mmol}, 0.471 \mathrm{~g}, 77 \%) .{ }^{1} \mathrm{H}$ NMR $(400 \mathrm{MHz}$, $\left.\mathrm{CDCl}_{3}\right) \delta 7.58-7.03(\mathrm{~m}, 14 \mathrm{H}), 4.64(\mathrm{dt}, J=9.1,3.3 \mathrm{~Hz}, 1 \mathrm{H}), 3.69(\mathrm{~s}, 2 \mathrm{H}), 2.91(\mathrm{~d}, J=2.7 \mathrm{~Hz}$, 1H), $2.79-2.59(\mathrm{~m}, 2 \mathrm{H}) ;{ }^{13} \mathrm{C}$ NMR $\left(101 \mathrm{MHz}, \mathrm{CDCl}_{3}\right) \delta 142.6,138.0,129.0,128.7,128.6$, 127.9, 127.3, 125.9, 71.8, 40.9, 36.2; HRMS (ESI) calcd for $\mathrm{C}_{15} \mathrm{H}_{16} \mathrm{OSNa}^{+}[\mathrm{M}+\mathrm{Na}]^{+} 267.0820$, found $267.0827 .{ }^{3}$

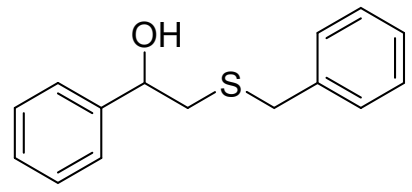

SI-4

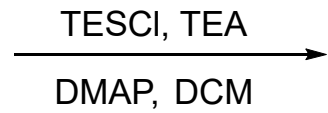

DMAP, DCM

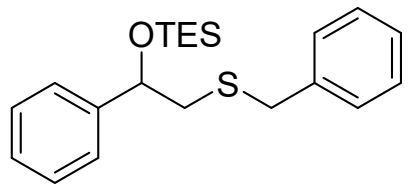

SI-5

Compound SI-5. A $10 \mathrm{~mL}$ two-neck flame-dried round-bottom flask was charged with SI-4 $(1.50 \mathrm{mmol}, 0.37 \mathrm{~g}, 1.0 \mathrm{eq})$ in dichloromethane $(5 \mathrm{~mL})$. Triethylamine $(1.65 \mathrm{mmol}, 0.23 \mathrm{~mL}, 1.1$ eq) and DMAP (0.075 mmol, $0.009 \mathrm{~g}, 0.05 \mathrm{eq})$ were added prior to dropwise addition of TESCl (1.50 mmol, $0.25 \mathrm{~mL}, 1.0 \mathrm{eq})$. After stirring for 4 hours, the reaction was diluted with dichloromethane $(40 \mathrm{~mL})$, washed $\left(3 \mathrm{x}, 10 \mathrm{~mL}\right.$, sat. $\left.\mathrm{Na}_{2} \mathrm{CO}_{3}\right)$ and $(1 \mathrm{x}, 10 \mathrm{~mL}$, brine), then dried with $\mathrm{MgSO}_{4}$, filtered, and concentrated in vacuo. The crude residue was purified by flash chroma- 
tography (20\% dichloromethane/hexanes) to yield SI-5 as a clear/colorless oil $(1.4 \mathrm{mmol}, 0.52$ g, 96\%). ${ }^{1} \mathrm{H}$ NMR (400 MHz, $\left.\mathrm{CDCl}_{3}\right) \delta 7.39-7.18(\mathrm{~m}, 8 \mathrm{H}), 4.69(\mathrm{dd}, J=7.4,5.4 \mathrm{~Hz}, 1 \mathrm{H}), 3.59$ (q, $J=13.3 \mathrm{~Hz}, 2 \mathrm{H}), 2.80-2.2 .55(\mathrm{~m}, 2 \mathrm{H}), 0.87(\mathrm{t}, J=7.9 \mathrm{~Hz}, 9 \mathrm{H}), 0.63-0.42(\mathrm{~m}, 6 \mathrm{H}) ;{ }^{13} \mathrm{C}$ NMR (101 MHz, $\left.\mathrm{CDCl}_{3}\right) \delta 144.3,138.8,129.2,128.6,128.3,127.7,127.1,126.3,75.5,41.4$, 37.2, 6.9, 5.0; El-GCMS m/z (in ACN): [M] ${ }^{+} \mathrm{C}_{21} \mathrm{H}_{30} \mathrm{OSSi}$ calcd 358.2, found 358 .

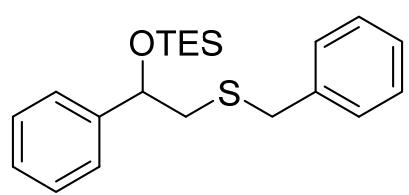

SI-5

$$
\underset{\mathrm{mCPBA},-78^{\circ} \mathrm{C}}{\mathrm{DCM}}
$$

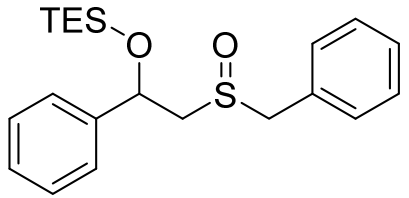

14

Compound 14. A $25 \mathrm{~mL}$ round-bottom flask was charged with dichloromethane $(6 \mathrm{~mL})$ and $\mathbf{S I}$ $5(0.20 \mathrm{mmol}, 0.072 \mathrm{~g}, 1.0 \mathrm{eq})$ prior to cooling to $-78^{\circ} \mathrm{C}$. mCPBA $(\sim 70 \%$ pure, $0.18 \mathrm{mmol}$, $0.043 \mathrm{~g}, 0.9 \mathrm{eq})$ dissolved in dichloromethane $(2 \mathrm{~mL})$ was then added by syringe pump over 30 minutes. The reaction stirred for 3 hours at $-78^{\circ} \mathrm{C}$ before warming and addition of saturated $\mathrm{Na}_{2} \mathrm{~S}_{2} \mathrm{O}_{3}(5 \mathrm{~mL})$. The organic phase was diluted with dichloromethane $(50 \mathrm{~mL})$, washed $(3 \mathrm{x}, 15$ $\mathrm{mL}$, sat. $\left.\mathrm{NaHCO}_{3}\right)$ and $\left(1 \mathrm{x}, 10 \mathrm{~mL}\right.$, brine), dried with $\mathrm{MgSO}_{4}$, filtered, and concentrated in vacuo. The crude material was purified by flash chromatography ( $25 \%$ ethyl acetate/hexanes) to yield 14 as a clear/colorless oil $(0.15 \mathrm{mmol}, 0.055 \mathrm{~g}, 82 \%) .{ }^{1} \mathrm{H}$ NMR $\left(400 \mathrm{MHz}, \mathrm{CDCl}_{3}\right) \delta 7.53$ - $7.28(\mathrm{~m}, 10 \mathrm{H}), 5.32(\mathrm{dd}, J=9.9,2.7 \mathrm{~Hz}, 1 \mathrm{H}), 4.48(\mathrm{~d}, J=13.7 \mathrm{~Hz}, 1 \mathrm{H}), 4.21(\mathrm{~d}, J=13.7 \mathrm{~Hz}$, 1H), $3.44(\mathrm{dd}, J=15.1,9.9 \mathrm{~Hz}, 1 \mathrm{H}), 2.85-2.73(\mathrm{~m}, 1 \mathrm{H}), 0.91(\mathrm{t}, J=7.9 \mathrm{~Hz}, 9 \mathrm{H}), 0.66-0.47$ $(\mathrm{m}, 6 \mathrm{H}) ;{ }^{13} \mathrm{C} \operatorname{NMR}\left(151 \mathrm{MHz}, \mathrm{CDCl}_{3}\right) \delta 142.1,131.3,129.1,129.0,128.9,128.7,128.6,126.3$, 71.4, 61.1, 59.5, 6.8, 4.9; HRMS (ESI) calcd for $\mathrm{C}_{21} \mathrm{H}_{31} \mathrm{O}_{2} \mathrm{SSi}^{+}[\mathrm{M}+\mathrm{H}]^{+} 375.1809$, found 375.1821.

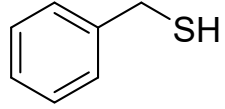

SI-6

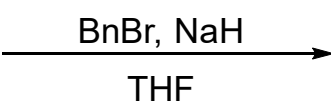

THF

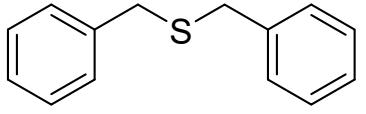

SI-7 
Compound SI-7. ${ }^{1} \mathrm{H}$ NMR (400 MHz, $\left.\mathrm{CDCl}_{3}\right) \delta 7.39-7.23(\mathrm{~m}, 10 \mathrm{H}), 3.63(\mathrm{~s}, 4 \mathrm{H}) ;{ }^{13} \mathrm{C} \mathrm{NMR}$ $\left(101 \mathrm{MHz}, \mathrm{CDCl}_{3}\right) \delta 138.3,129.2,128.6,127.1,35.7 .{ }^{4}$

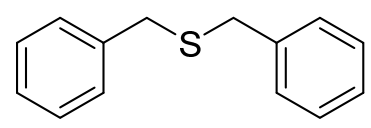

SI-7

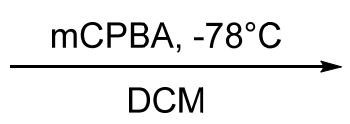

DCM

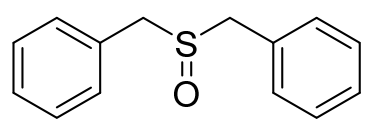

SI-8

Compound SI-8. A $250 \mathrm{~mL}$ round-bottom flask was charged with SI-7 $(2.0 \mathrm{mmol}, 0.43 \mathrm{~g}, 1.0$ eq) and dichloromethane $(60 \mathrm{~mL})$. After cooling the flask to $-78^{\circ} \mathrm{C}$, a solution of $\mathrm{mCPBA}(\sim 70 \%$ pure, $1.8 \mathrm{mmol}, 0.44 \mathrm{~g}, 0.9 \mathrm{eq})$ in dichloromethane $(20 \mathrm{~mL})$ was added via syringe pump over 30 min. After stirring for 3 hours the reaction was warmed and quenched with a saturated $\mathrm{Na}_{2} \mathrm{~S}_{2} \mathrm{O}_{3}$ solution $(5 \mathrm{~mL})$. The organic phase was washed $\left(3 \mathrm{x}, 20 \mathrm{~mL}\right.$, sat. $\left.\mathrm{NaHCO}_{3}\right)$ and $(1 \mathrm{x}$, $10 \mathrm{~mL}$, brine), dried with $\mathrm{MgSO}_{4}$, filtered, and concentrated in vacuo. The material was quite pure and used without further purification. (white solid) ${ }^{1} \mathrm{H}$ NMR $\left(600 \mathrm{MHz}, \mathrm{CDCl}_{3}\right) \delta 7.44-$ $7.27(\mathrm{~m}, 10 \mathrm{H}), 3.93$ and $3.88(\mathrm{AB} \mathrm{q}, J=13.0,4 \mathrm{H}) ;{ }^{13} \mathrm{C} \mathrm{NMR}\left(151 \mathrm{MHz}, \mathrm{CDCl}_{3}\right) \delta 130.3,130.3$, $129.1,128.5,57.4 .^{5}$

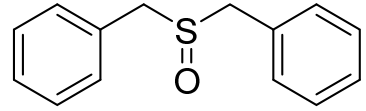

SI-8

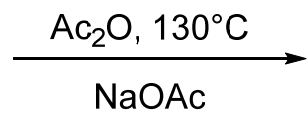

$\mathrm{NaOAc}$

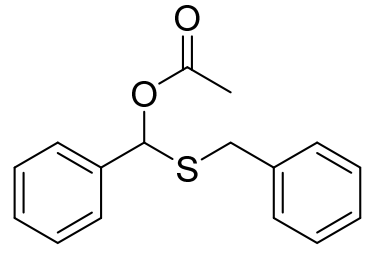

SI-9

Compound SI-9. A round-bottom flask was charged with SI-8 $(0.90 \mathrm{mmol}, 0.21 \mathrm{~g}, 1.0 \mathrm{eq})$ and freshly distilled acetic anhydride $(8 \mathrm{~mL})$. Anhydrous sodium acetate $(0.27 \mathrm{mmol}, 0.037 \mathrm{~g}, 0.3$ eq) was added to the solution and the reaction heated to reflux for 4 hours. The reaction was then concentrated in vacuo at $75^{\circ} \mathrm{C}$ and taken up in ethyl acetate $(60 \mathrm{~mL})$. The organic phase was then washed $\left(3 \mathrm{x}, 20 \mathrm{~mL}\right.$, sat. $\left.\mathrm{NaHCO}_{3}\right)$ and $\left(1 \mathrm{x}, 10 \mathrm{~mL}\right.$, brine). After drying with $\mathrm{MgSO}_{4}$ the organic phase was concentrated in vacuo to yield SI-9 as a brown oil $(0.82 \mathrm{mmol}, 0.22 \mathrm{~g}$, 
91\%). ${ }^{1} \mathrm{H}$ NMR (400 MHz, $\left.\mathrm{CDCl}_{3}\right) \delta 7.43-7.22(\mathrm{~m}, 10 \mathrm{H}), 6.91(\mathrm{~s}, 1 \mathrm{H}), 3.92$ and 3.79 (AB q, J $=13.4,2 \mathrm{H}), 2.04(\mathrm{~s}, 3 \mathrm{H}) ;{ }^{13} \mathrm{C} \mathrm{NMR}\left(101 \mathrm{MHz}, \mathrm{CDCl}_{3}\right) \delta 170.1,137.8,137.8,129.1,128.7$, 128.7, 128.6, 127.3, 126.5, 79.4, 36.2, 21.2; ESI-MS m/z $[\mathrm{M}+\mathrm{H}]^{+} \mathrm{C}_{16} \mathrm{H}_{17} \mathrm{O}_{2} \mathrm{~S}$ calcd 273.0, found 273; [M+Na] ${ }^{+} \mathrm{C}_{16} \mathrm{H}_{16} \mathrm{NaO}_{2} \mathrm{~S}$ calcd 295.1, found 295.

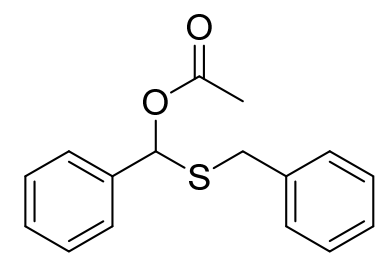

SI-9
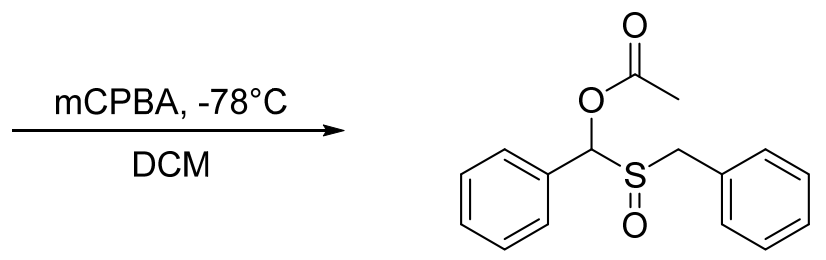

15

Compound 15. A $25 \mathrm{~mL}$ round-bottom flask was charged with SI-9 $(0.20 \mathrm{mmol}, 0.055 \mathrm{~g}, 1.0$ eq) and dichloromethane $(6 \mathrm{~mL})$. After cooling the flask to $-78^{\circ} \mathrm{C}$, a solution of $\mathrm{mCPBA}(\sim 70 \%$ pure, $0.18 \mathrm{mmol}, 0.044 \mathrm{~g}, 0.9 \mathrm{eq})$ in dichloromethane $(2 \mathrm{~mL})$ was added via syringe pump over $30 \mathrm{~min}$. After 3 hours stirring at $-78^{\circ} \mathrm{C}$, the reaction was warmed to room temperature and saturated $\mathrm{Na}_{2} \mathrm{~S}_{2} \mathrm{O}_{3}(5 \mathrm{~mL})$ was added. The organic phase was separated, diluted with dichloromethane $(50 \mathrm{~mL})$, and washed $\left(3 \mathrm{x}, 15 \mathrm{~mL}\right.$, sat. $\left.\mathrm{NaHCO}_{3}\right)$ and $(1 \mathrm{x}, 10 \mathrm{~mL}$, brine). It was then dried with $\mathrm{MgSO}_{4}$, filtered, and concentrated in vacuo. The crude material was purified by flash chromatography ( $40 \%$ ethyl acetate/hexanes) to yield $15(0.16 \mathrm{mmol}, 0.045 \mathrm{~g}, 87 \%)$ as a mix of diastereomers, appearing as a viscous hazy golden oil. ${ }^{1} \mathrm{H} \mathrm{NMR}\left(400 \mathrm{MHz}, \mathrm{CDCl}_{3}\right) \delta 7.52-$ $7.39(\mathrm{~m}, 5 \mathrm{H}), 7.39-7.29(\mathrm{~m}, 3 \mathrm{H}), 7.25-7.18(\mathrm{~m}, 2 \mathrm{H}), 6.63-6.53(\mathrm{~s}, 1 \mathrm{H}), 3.89-3.66(\mathrm{~m}$, 2H), 2.24 (s, 3H); ${ }^{13} \mathrm{C}$ NMR (101 MHz, $\left.\mathrm{CDCl}_{3}\right) \delta 169.2,169.2,131.3,130.3,130.2,130.2$, $130.2,130.1,130.1,130.0,129.2,129.1,128.9,128.6,128.5,128.0,127.9,87.0,86.7,55.1$, 54.7, 20.9, 20.9; HRMS (ESI) calcd for $\mathrm{C}_{16} \mathrm{H}_{17} \mathrm{O}_{3} \mathrm{~S}^{+}[\mathrm{M}+\mathrm{H}]^{+}$289.0893, found 289.0890 .

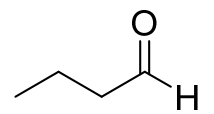

SI-1

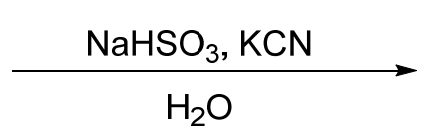

$\mathrm{H}_{2} \mathrm{O}$<smiles>CCCC(O)C#N</smiles>

SI-10 
Compound SI-10. ${ }^{1} \mathrm{H}$ NMR (600 MHz, $\left.\mathrm{CDCl}_{3}\right) \delta 4.48(\mathrm{t}, J=6.8 \mathrm{~Hz}, 1 \mathrm{H}), 2.70(\mathrm{~s}, 1 \mathrm{H}), 1.95-$ $1.75(\mathrm{~m}, 2 \mathrm{H}), 1.54(\mathrm{~h}, J=7.4 \mathrm{~Hz}, 2 \mathrm{H}), 0.99(\mathrm{t}, J=7.4 \mathrm{~Hz}, 3 \mathrm{H}) ;{ }^{13} \mathrm{C} \mathrm{NMR}\left(151 \mathrm{MHz}, \mathrm{CDCl}_{3}\right) \delta$ $120.3,60.9,37.0,17.9,13.4 .^{6}$

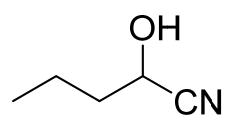

SI-10

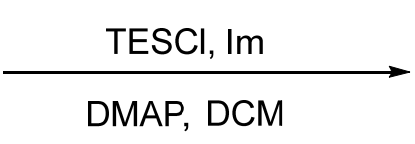

DMAP, DCM

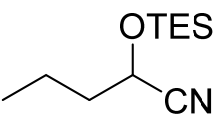

16

Compound 16. A $100 \mathrm{~mL}$ two-neck flame-dried round-bottom flask was charged with dichloromethane $(60 \mathrm{~mL})$ and $\mathbf{S I}-10(12.0 \mathrm{mmol}, 1.00 \mathrm{~g}, 1.0 \mathrm{eq})$ before cooling to $0^{\circ} \mathrm{C}$. After addition of imidazole (24.0 mmol, $1.63 \mathrm{~g}, 2.0 \mathrm{eq})$, DMAP (0.6 mmol, $0.073 \mathrm{~g}, 0.05 \mathrm{eq})$, and TESCl (14.4 $\mathrm{mmol}, 2.17 \mathrm{~g}, 1.2 \mathrm{eq}$ ) the reaction was stirred for $20 \mathrm{~min}$ at $0^{\circ} \mathrm{C}$ followed by 6 hours at $25^{\circ} \mathrm{C}$. The organic phase was washed $\left(1 \mathrm{x}, 30 \mathrm{~mL}, \mathrm{H}_{2} \mathrm{O}\right)$ and $(1 \mathrm{x}, 30 \mathrm{~mL}$, brine $)$ then dried with $\mathrm{MgSO}_{4}$, filtered, and concentrated in vacuo. The crude oil was then purified by flash chromatography (5\% ethyl acetate/hexanes) to yield 16 as a clear/gold tinted oil $(8.8 \mathrm{mmol}, 1.87 \mathrm{~g}$, 73\%). ${ }^{1} \mathrm{H}$ NMR $\left(600 \mathrm{MHz}, \mathrm{CDCl}_{3}\right) \delta 4.43(\mathrm{t}, \mathrm{J}=6.5 \mathrm{~Hz}, 1 \mathrm{H}), 1.80-1.74(\mathrm{~m}, 2 \mathrm{H}), 1.55-1.46$ $(\mathrm{m}, 2 \mathrm{H}), 1.01-0.93(\mathrm{~m}, 12 \mathrm{H}), 0.74-0.61(\mathrm{~m}, 6 \mathrm{H}) ;{ }^{13} \mathrm{C} \mathrm{NMR}\left(151 \mathrm{MHz}, \mathrm{CDCl}_{3}\right) \delta$ 120.3, 61.7, 38.6, 18.0, 13.6, 6.7, 4.5; El-GCMS $m / z$ (in ACN): $[M]^{+} \mathrm{C}_{11} \mathrm{H}_{23} \mathrm{NOSi}$ calcd 213.1, found 213; $[\mathrm{M}+\mathrm{H}]^{+} \mathrm{C}_{11} \mathrm{H}_{24} \mathrm{NOSi}$ calcd 214.1, found 214 .

\section{Stability studies of control compounds $(14,15,16)$}

Each control compound was subjected to mCPBA oxidation conditions as described above. The progress of the reaction was monitored by TLC and we did not observe any significant change to the control compound after $3 \sim 5$ hours. After the reaction, the control compound was recovered (>95\%) from the reaction mixture by extraction and confirmed by NMR analysis.

\section{Procedures for oxidation-triggered allicin formation}


<smiles>[R]C(O[Si])SCC=C</smiles>

17a: $\mathrm{R}=\mathrm{nPr}, \mathrm{Si}=\mathrm{TMS}$

17b: $\mathrm{R}=\mathrm{nPr}, \mathrm{Si}=\mathrm{TES}$

17c: $R=i B u, S i=$ TES

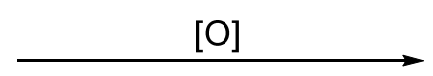

Allicin

\section{mCPBA Oxidation:}

Formation of Allicin. A round-bottom flask was charged with $17 \mathrm{a}(0.40 \mathrm{mmol}, 0.087 \mathrm{~g}, 1.0 \mathrm{eq})$ and dichloromethane $(12 \mathrm{~mL})$. After cooling the flask to $-78^{\circ} \mathrm{C}$, a solution of mCPBA $(\sim 70 \%$ pure, $0.36 \mathrm{mmol}, 0.089 \mathrm{~g}, 0.9 \mathrm{eq})$ in dichloromethane $(4 \mathrm{~mL})$ was added via syringe pump over 30 min. After 3 hours the reaction was warmed to $25^{\circ} \mathrm{C}$, quenched with sat. $\mathrm{Na}_{2} \mathrm{~S}_{2} \mathrm{O}_{3}(5 \mathrm{~mL})$, and diluted with dichloromethane $(50 \mathrm{~mL})$. The organic phase was washed $(3 \mathrm{x}, 15 \mathrm{~mL}$, sat. $\left.\mathrm{NaHCO}_{3}\right)$, the resulting aqueous phase extracted $(2 x, 15 \mathrm{~mL}, \mathrm{DCM})$, and the combined organic phases washed $\left(1 \mathrm{x}, 10 \mathrm{~mL}\right.$, brine). After drying with $\mathrm{MgSO}_{4}$ and concentrating in vacuo, the crude material was purified by flash chromatography (15\% ethyl acetate/hexane) to yield allicin $(0.05 \mathrm{mmol}, 0.008 \mathrm{~g}, 29 \%)$ as a clear/light yellow oil. ${ }^{1} \mathrm{H}$ NMR $\left(400 \mathrm{MHz}, \mathrm{CDCl}_{3}\right) \delta 6.07-$ $5.78(\mathrm{~m}, 1 \mathrm{H}), 5.56-5.03(\mathrm{~m}, 2 \mathrm{H}), 4.04-3.71(\mathrm{~m}, 2 \mathrm{H}) .{ }^{13} \mathrm{C}$ NMR $\left(151 \mathrm{MHz}, \mathrm{CDCl}_{3}\right) \delta$ 133.0, $125.9,124.2,119.2,60.0,35.2 .^{2}$

Formation of Allicin. Substrate 17b. (0.4 mmol scale, $0.07 \mathrm{mmol}, 0.012 \mathrm{~g}, 40 \%)$ and $(1.2$ mmol scale, $0.35 \mathrm{mmol}, 0.056 \mathrm{~g}, 64 \%)$.

Formation of Allicin. Substrate 17c. (0.13 mmol, $0.021 \mathrm{~g}, 71 \%)$.

\section{$\underline{\mathrm{H}_{2} \mathrm{O}_{2}}$ Oxidation:}

Formation of Allicin. Two $4 \mathrm{~mL}$ vials were charged with $\mathrm{H}_{2} \mathrm{O}_{2}(0.98 \mathrm{M}, 1.28 \mathrm{~mL}, 25.0$ eq) and $\mathrm{H}_{2} \mathrm{O}(0.12 \mathrm{~mL}) .17 \mathrm{a}(0.05 \mathrm{mmol}, 0.011 \mathrm{~g}, 1.0 \mathrm{eq})$ was dissolved in THF $(0.6 \mathrm{~mL})$ and combined with the aq. $\mathrm{H}_{2} \mathrm{O}_{2}$ to make a $\mathrm{H}_{2} \mathrm{O} / \mathrm{THF}(70 / 30)$ solution $(2 \mathrm{~mL})$. The vials were sealed and heated to $37^{\circ} \mathrm{C}$ for $30 \mathrm{~min}$ and 4 hours, respectively. The solutions were extracted $(3 \mathrm{x}, 0.7 \mathrm{~mL}$, $\mathrm{DCM}$ ) and organic phases were dried with $\mathrm{MgSO}_{4}$. After filtration via a $0.2 \mu \mathrm{m}$ filter the solu- 
tions were concentrated gently. The crude material was then taken up in acetonitrile $(1 \mathrm{~mL})$ and diluted for quantitation by HPLC with external standard to yield allicin. The corresponding yields were noted in Table 1. The yields were determined by HPLC using a Supelcosil ${ }^{\mathrm{TM}}$ LC-18 column $(50 \times 4.6 \mathrm{~mm}, 5 \mu \mathrm{m}), \mathrm{ACN} / \mathrm{H}_{2} \mathrm{O}=5 / 95$ to $95 / 5$ gradient, flow rate $=2.0 \mathrm{~mL} / \mathrm{min}, \mathrm{I}=214$ $\mathrm{nm} ; \mathrm{t}_{\mathrm{R}}=3.0 \mathrm{~min}$ (major). The purified allicin HPLC fraction was collected and confirmed by ESI-MS $m / z[M+H]^{+} \mathrm{C}_{6} \mathrm{H}_{11} \mathrm{~S}_{2}$ calcd 163.0, found 163.

Reaction:

17a with $\mathrm{H}_{2} \mathrm{O}_{2}$ at 30 min:

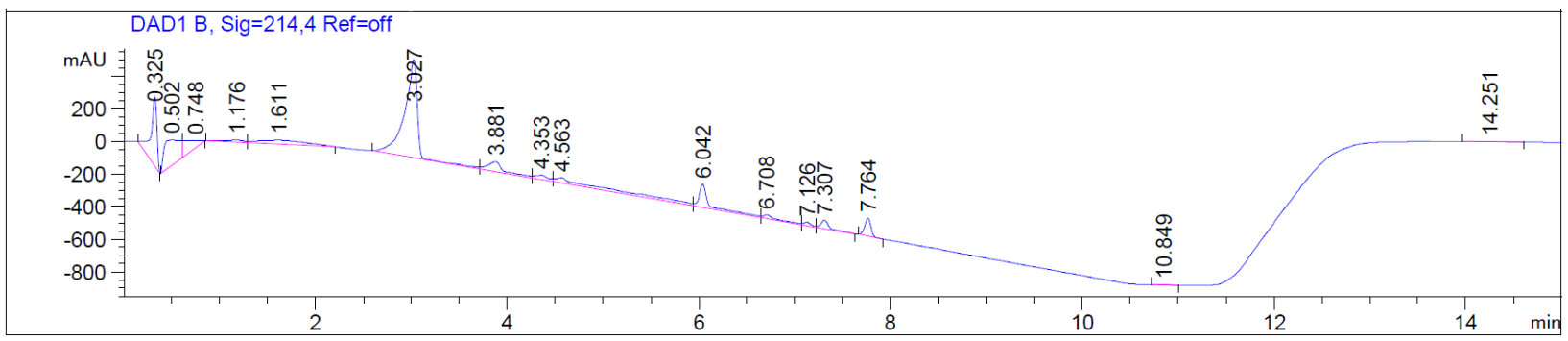

17a with $\mathrm{H}_{2} \mathrm{O}_{2}$ at $4 \mathrm{hr}$ :

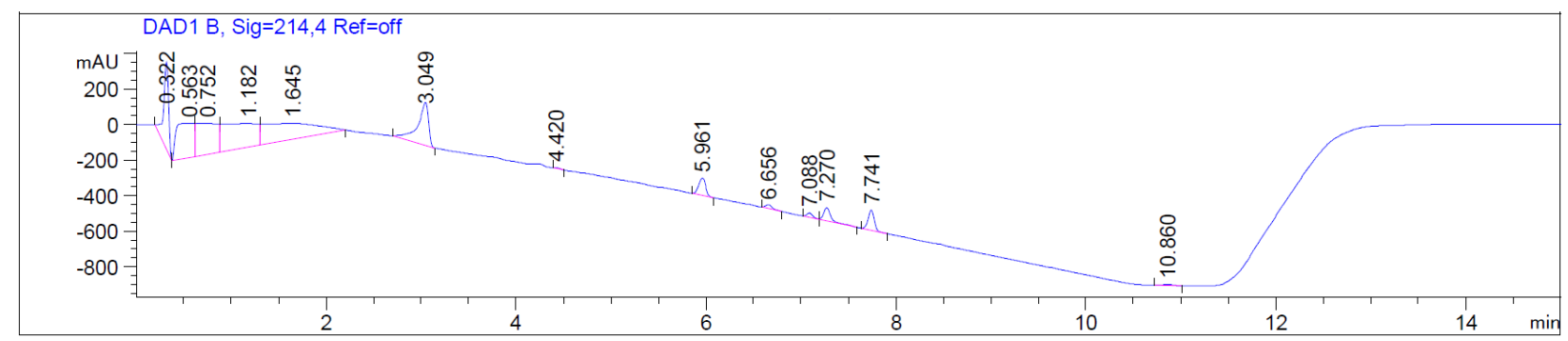

Allicin standard:

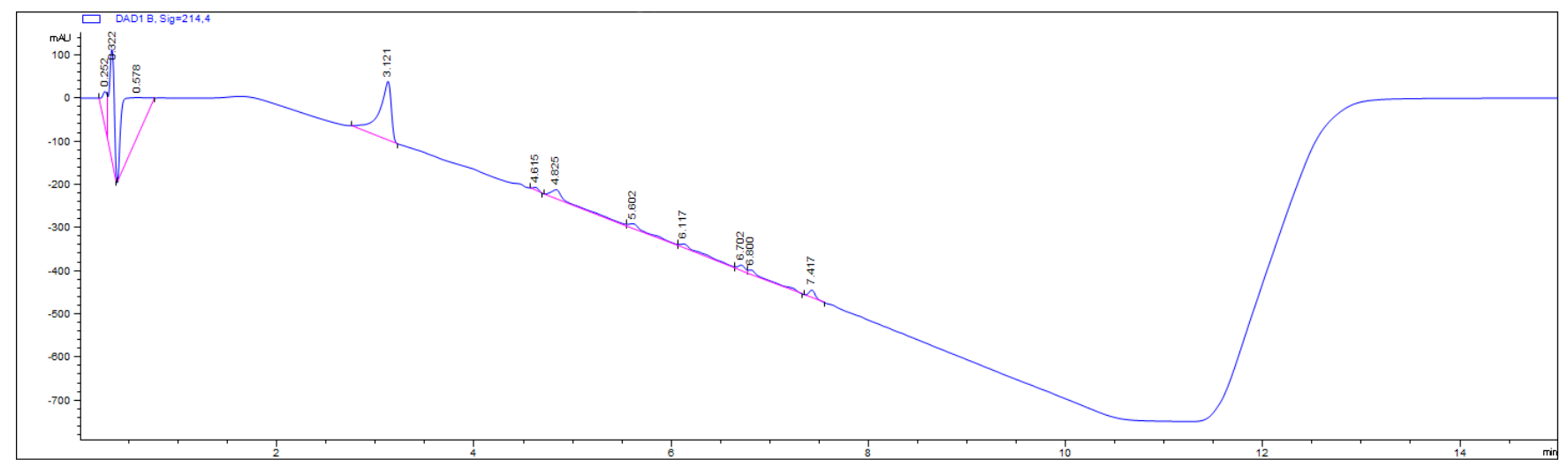

Starting material (17a) standard: 


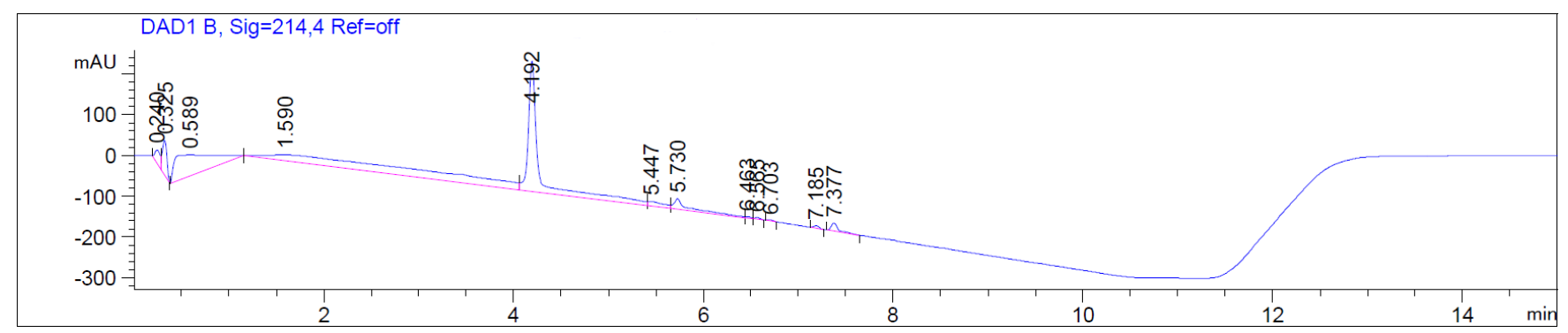

\section{NaOCl Oxidation:}

Formation of Allicin. A $4 \mathrm{~mL}$ vial was charged with $\mathrm{H}_{2} \mathrm{O}_{2}\left(0.98 \mathrm{M}, 1.28 \mathrm{~mL}, 25.0\right.$ eq) and $\mathrm{H}_{2} \mathrm{O}$ $(0.12 \mathrm{~mL}) .17 \mathrm{a}(0.05 \mathrm{mmol}, 0.011 \mathrm{~g}, 1.0 \mathrm{eq})$ was dissolved in THF $(0.6 \mathrm{~mL})$ and combined with the aq. $\mathrm{H}_{2} \mathrm{O}_{2}$ to make a $\mathrm{H}_{2} \mathrm{O} / \mathrm{THF}(70 / 30)$ solution $(2 \mathrm{~mL})$. The vial was sealed and heated to $37^{\circ} \mathrm{C}$ for 24 hours and monitored by TLC. The solution was extracted (3x, $\left.0.7 \mathrm{~mL}, \mathrm{DCM}\right)$, organic phase then washed ( $1 \mathrm{x}, 1 \mathrm{~mL}$, brine) and dried with $\mathrm{MgSO}_{4}$. After filtration via a $0.2 \mu \mathrm{m}$ filter the solution was concentrated gently. The crude material was then taken up in acetonitrile $(1 \mathrm{~mL})$ and diluted for quantitation by HPLC with external standard. No allicin was detected.

\section{MMPP Oxidation:}

Formation of Allicin. A $10 \mathrm{~mL}$ round-bottom flask was charged with $17 \mathrm{~b}(0.4 \mathrm{mmol}, 0.10 \mathrm{~g}, 1.0$ eq), tetrahydrofuran $(3.6 \mathrm{~mL})$, and $\mathrm{H}_{2} \mathrm{O}(0.4 \mathrm{~mL})$. The reaction was cooled to $0^{\circ} \mathrm{C}$ and magnesium monoperoxyphthalate ( $80 \%$ pure, $0.2 \mathrm{mmol}, 0.12 \mathrm{~g}, 0.5 \mathrm{eq})$ added portion wise. After 45 minutes the reaction was diluted with ethyl acetate $(60 \mathrm{~mL})$. The organic phase was then washed $\left(1 \mathrm{x}, 10 \mathrm{~mL}, \mathrm{H}_{2} \mathrm{O}\right)$ and $\left(3 \mathrm{x}, 10 \mathrm{~mL}\right.$, sat. $\left.\mathrm{NaHCO}_{3}\right)$. The combined aqueous phase was extracted (3x, $10 \mathrm{~mL}$, EtOAc). The combined organic phase then washed (1x, $10 \mathrm{~mL}$, brine), dried with $\mathrm{MgSO}_{4}$, filtered, and purified by flash chromatography (15\% ethyl acetate/hexanes) to yield allicin as a clear/light yellow oil $(0.14 \mathrm{mmol}, 0.024 \mathrm{~g}, 74 \%)$.

\section{$\mathrm{NalO}_{4}$ Oxidation:}

Formation of Allicin. A $25 \mathrm{~mL}$ round-bottom flask was charged with $17 \mathrm{~b}(0.4 \mathrm{mmol}, 0.10 \mathrm{~g}, 1.0$ eq), methanol $(20 \mathrm{~mL})$. The reaction was cooled to $0^{\circ} \mathrm{C}$ and aq. $\mathrm{NalO}_{4}(0.5 \mathrm{M}, 0.4 \mathrm{mmol}, 0.8$ 
$\mathrm{mL}, 1.0 \mathrm{eq}$ ) added dropwise. The reaction was monitored by TLC and did not produce allicin after 8 hours.

\section{References:}

1. A. Ionescu, M. Ruppel and O. F. Wendt, J. Organomet. Chem., 2006, 691, 3806-3815.

2. T. Waag, C. Gelhaus, J. Rath, A. Stich, M. Leippe and T. Schirmeister, Bioorg. Med. Chem. Lett., 2010, 20, 5541-5543.

3. J. Zhu, R. Li, Z. Ge, T. Cheng and R. Li, Chin. J. Chem., 2009, 27, 791-796.

4. S. Enthaler and M. Weidauer, Catal. Lett., 2011, 141, 833-838.

5. C. Boudou, M. Bergès, C. Sagnes, J. Sopková-de Oliveira Santos, S. Perrio and P. Metzner, J. Org. Chem., 2007, 72, 5403-5406.

6. N. P. Chandia, J. C. Canales, I. Azocar, S. Vanlaer, V. G. Pawar, W. M. De Borggraeve, J. Costamagna and W. Dehaen, Synth. Commun., 2009, 39, 927-939. 


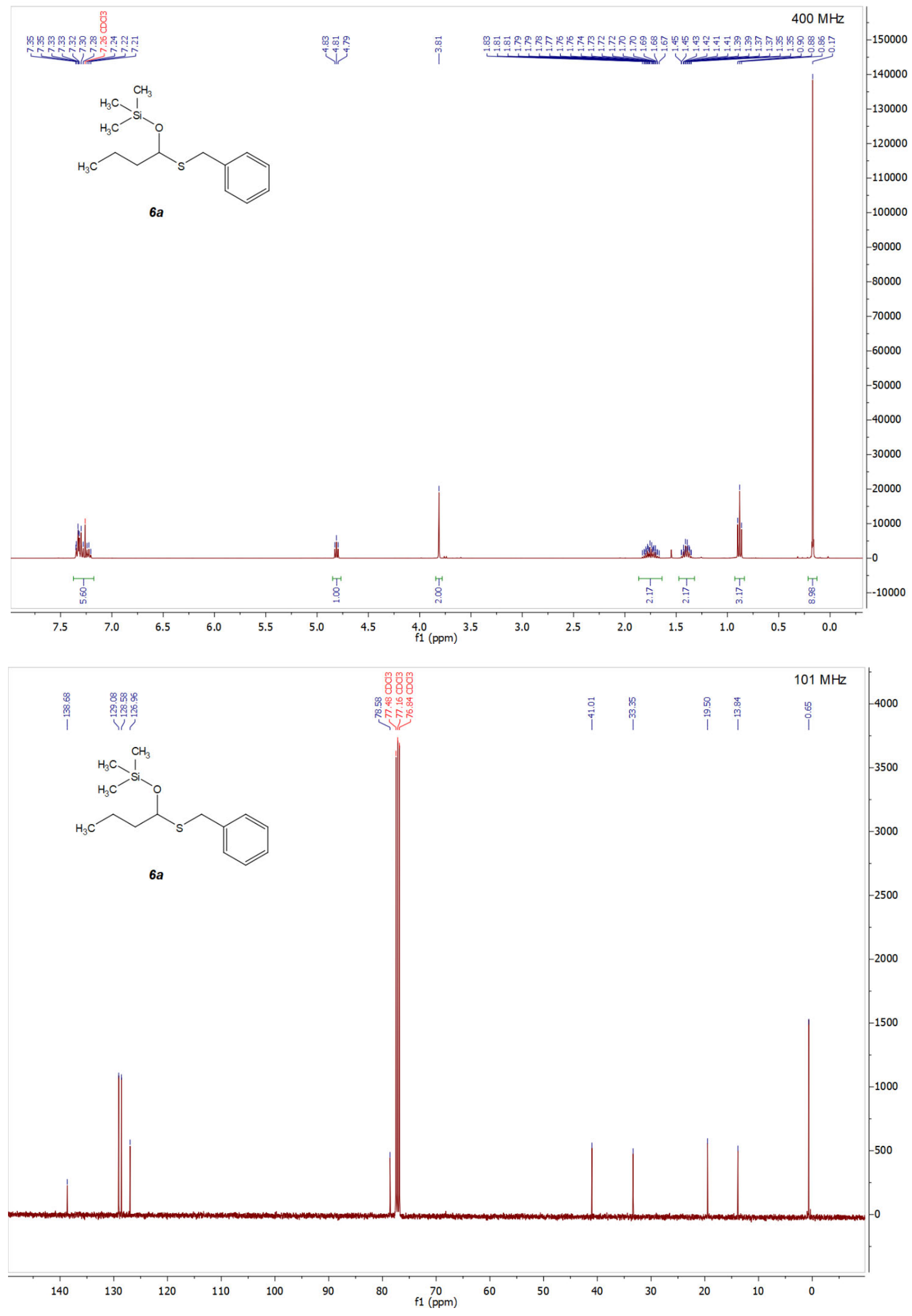



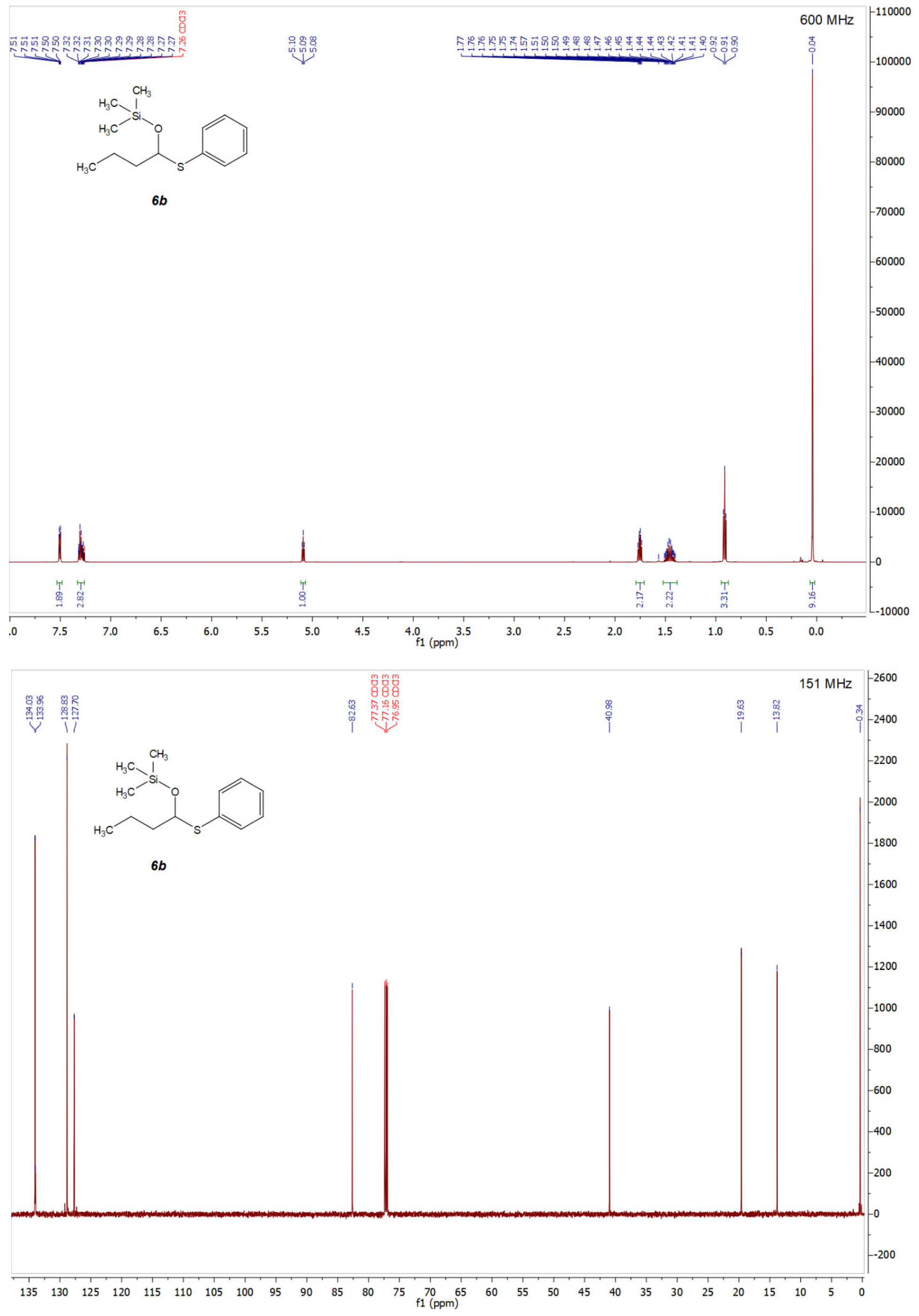


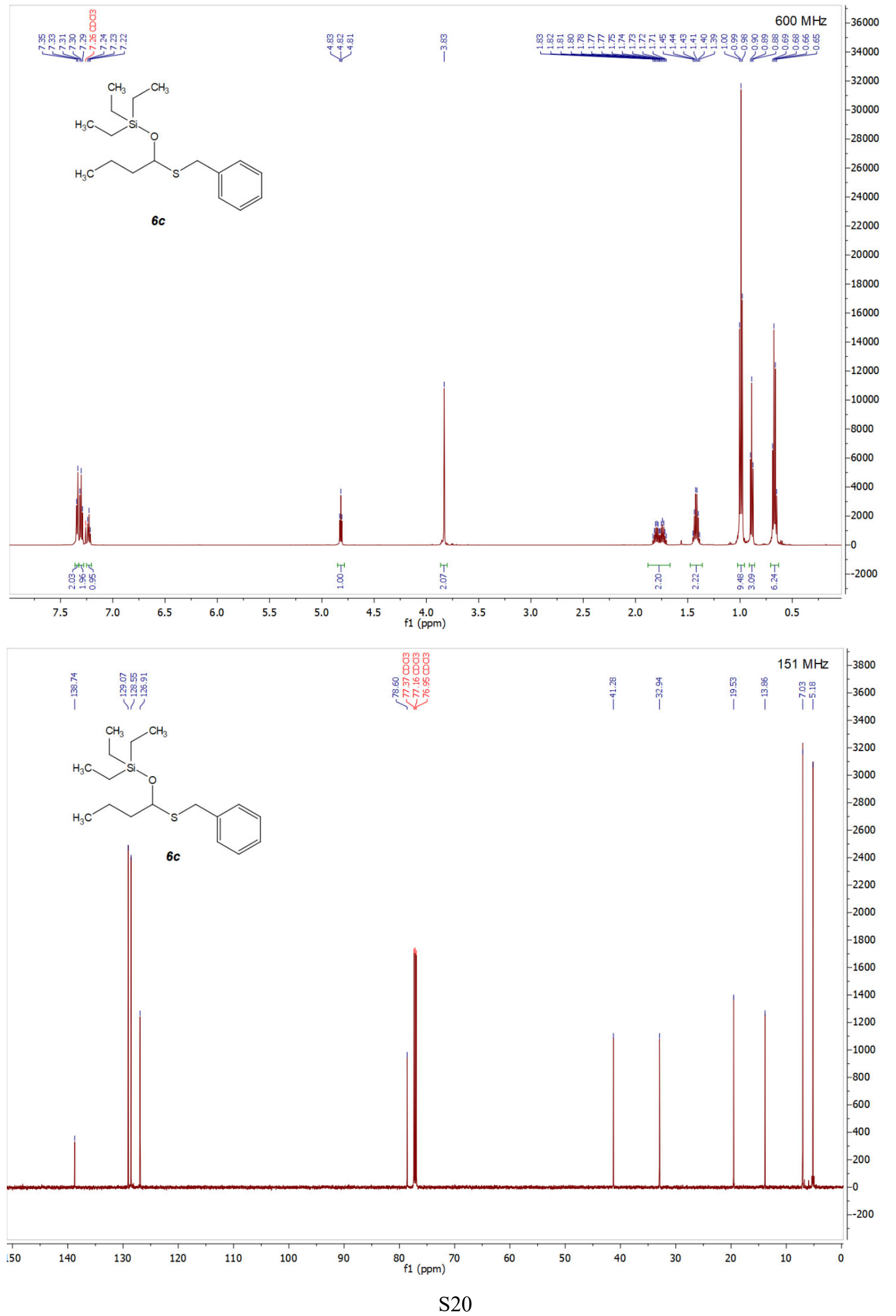




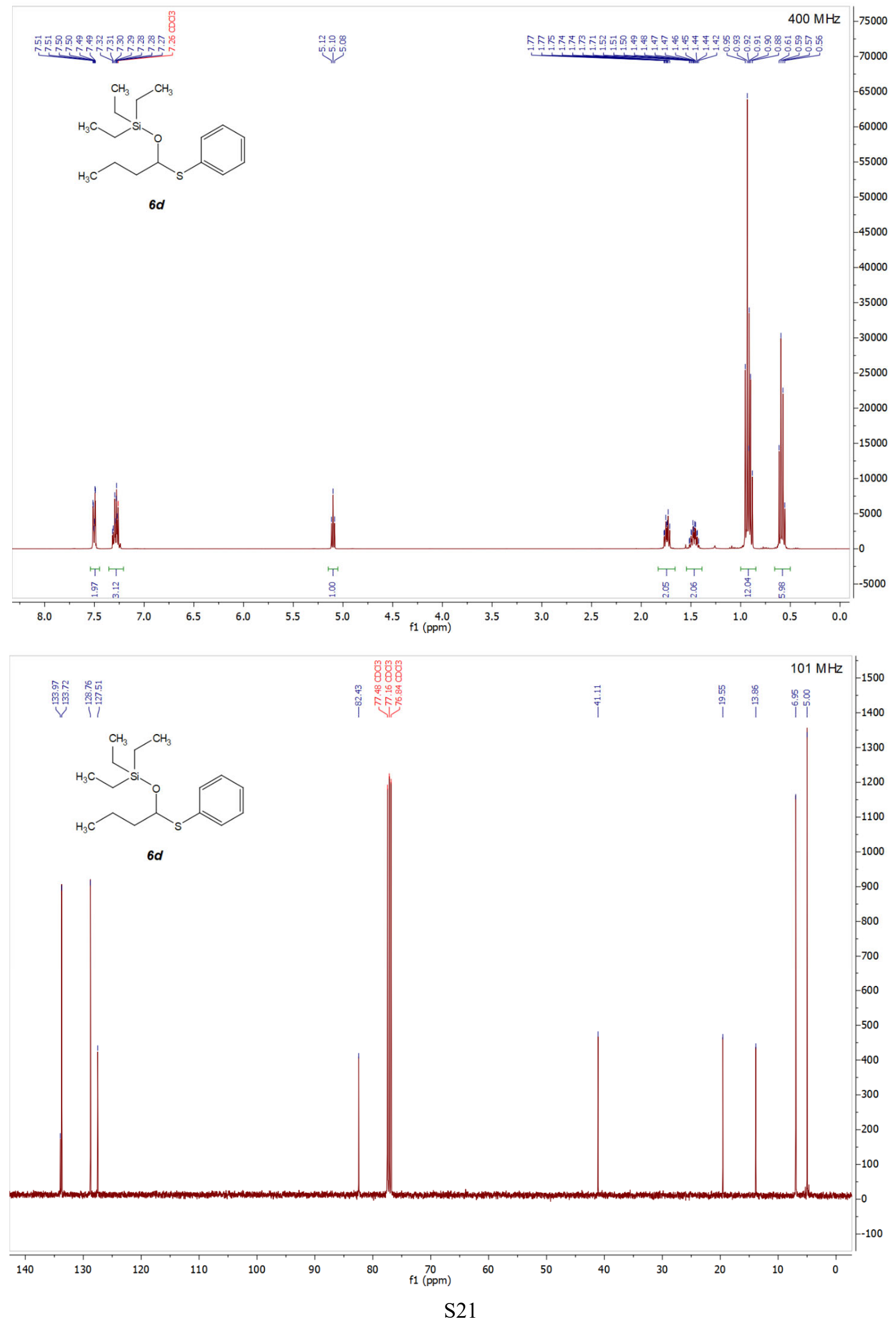




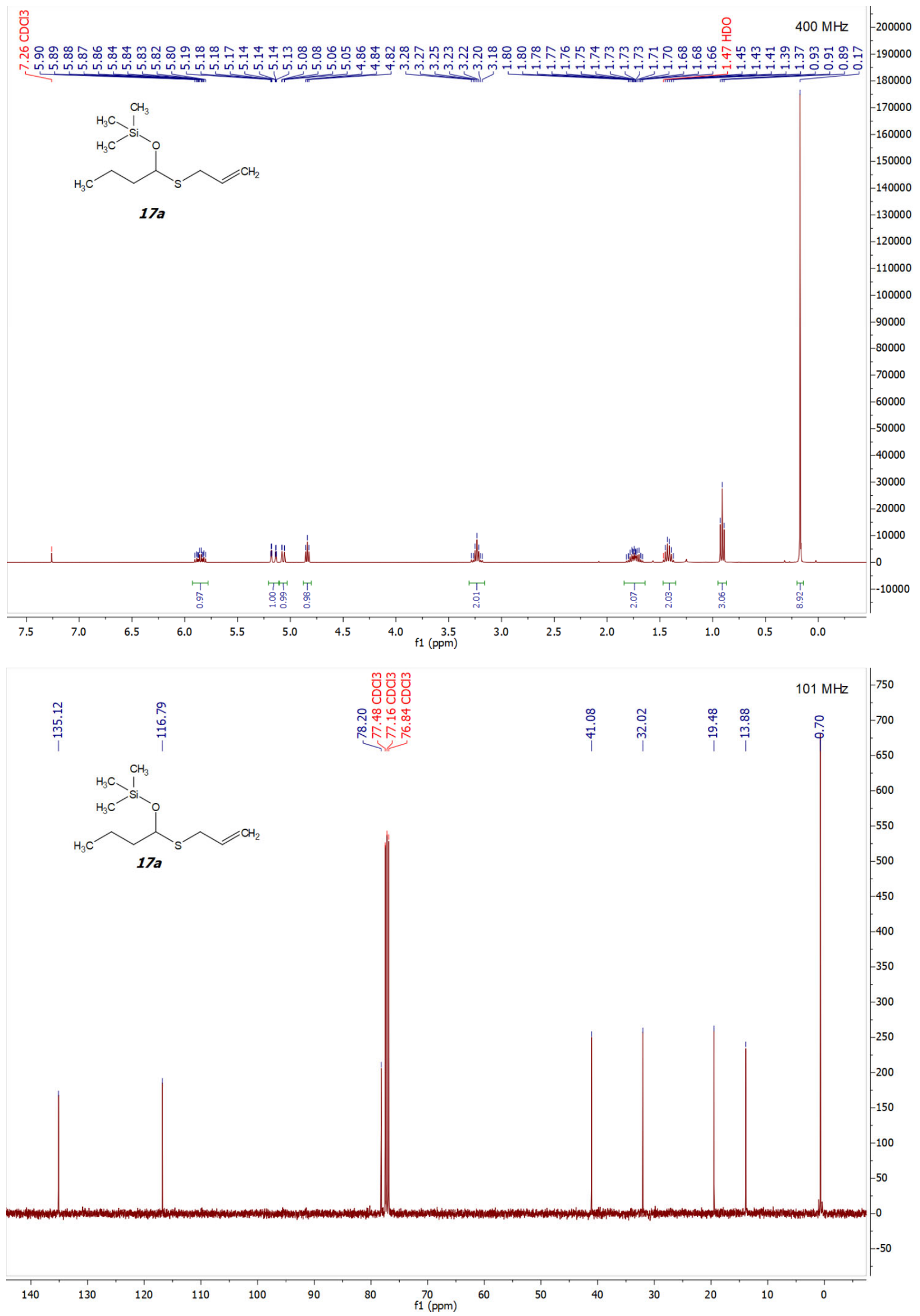




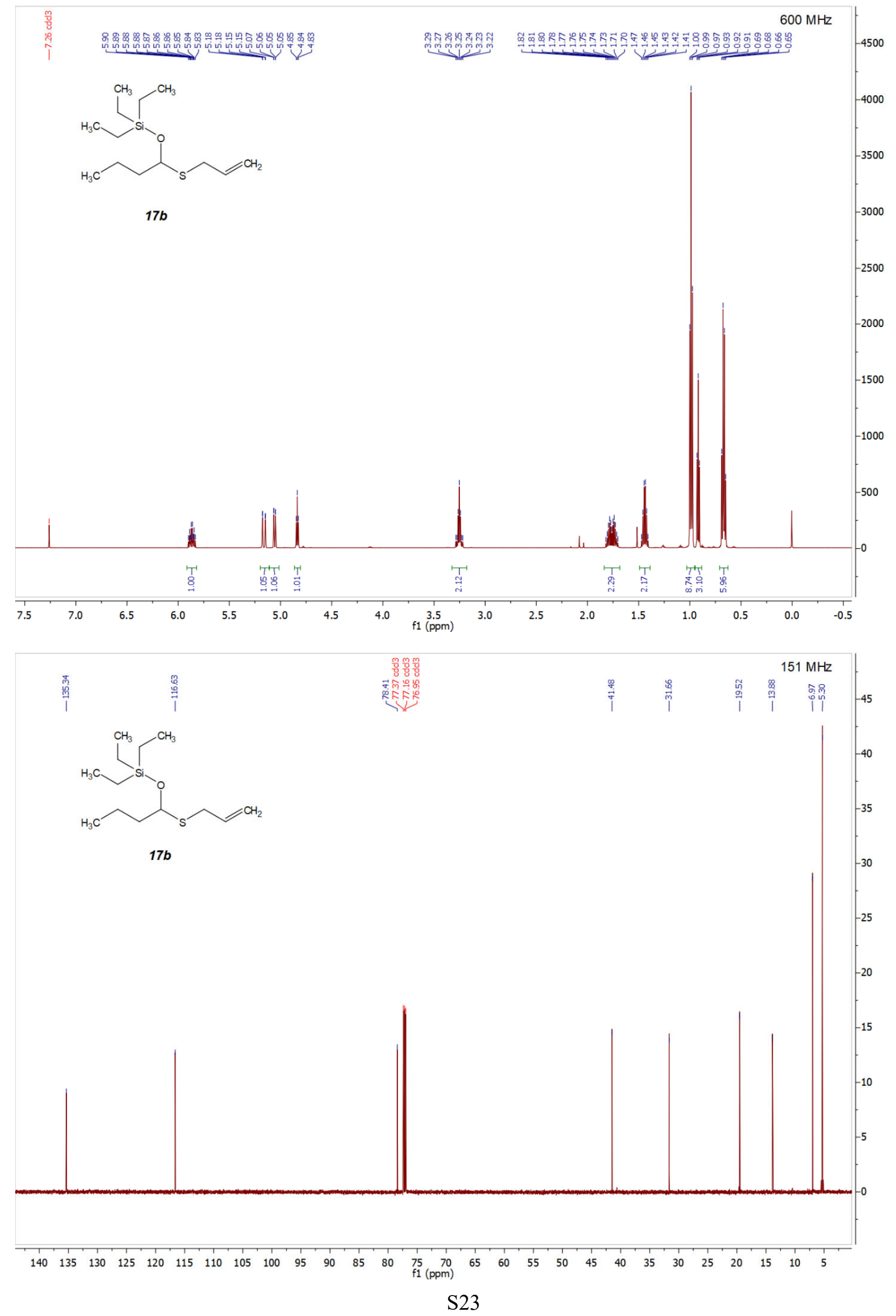




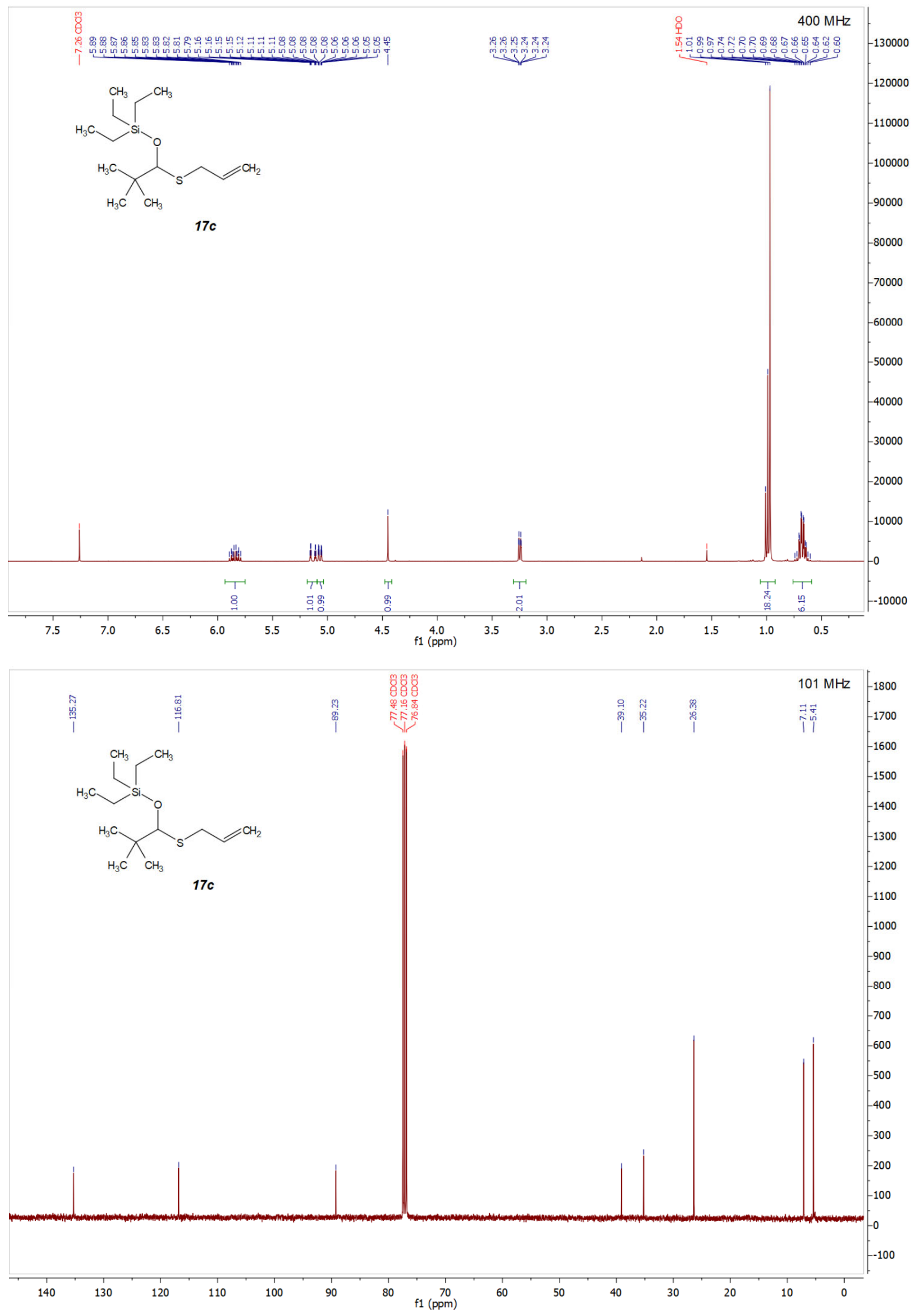




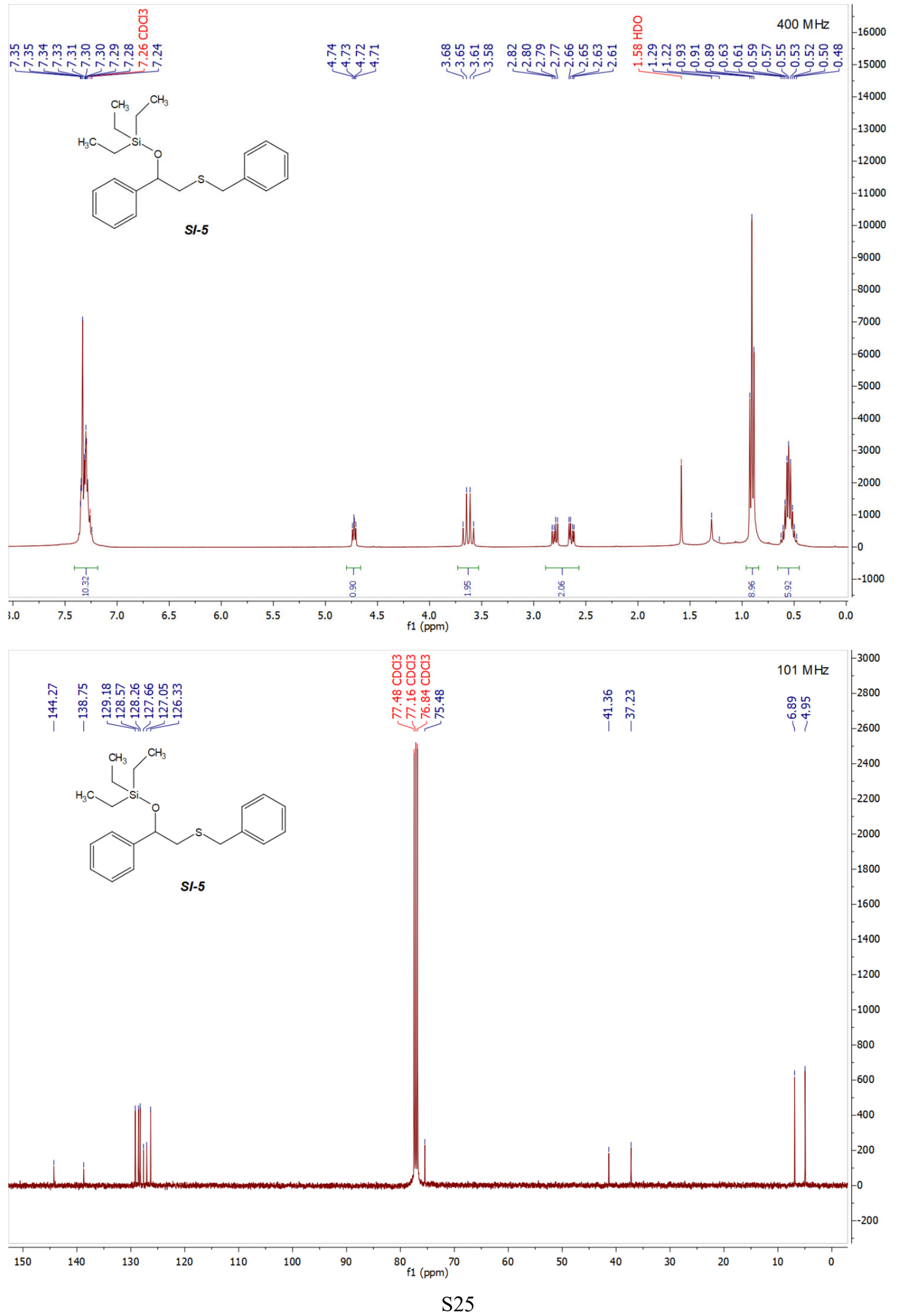




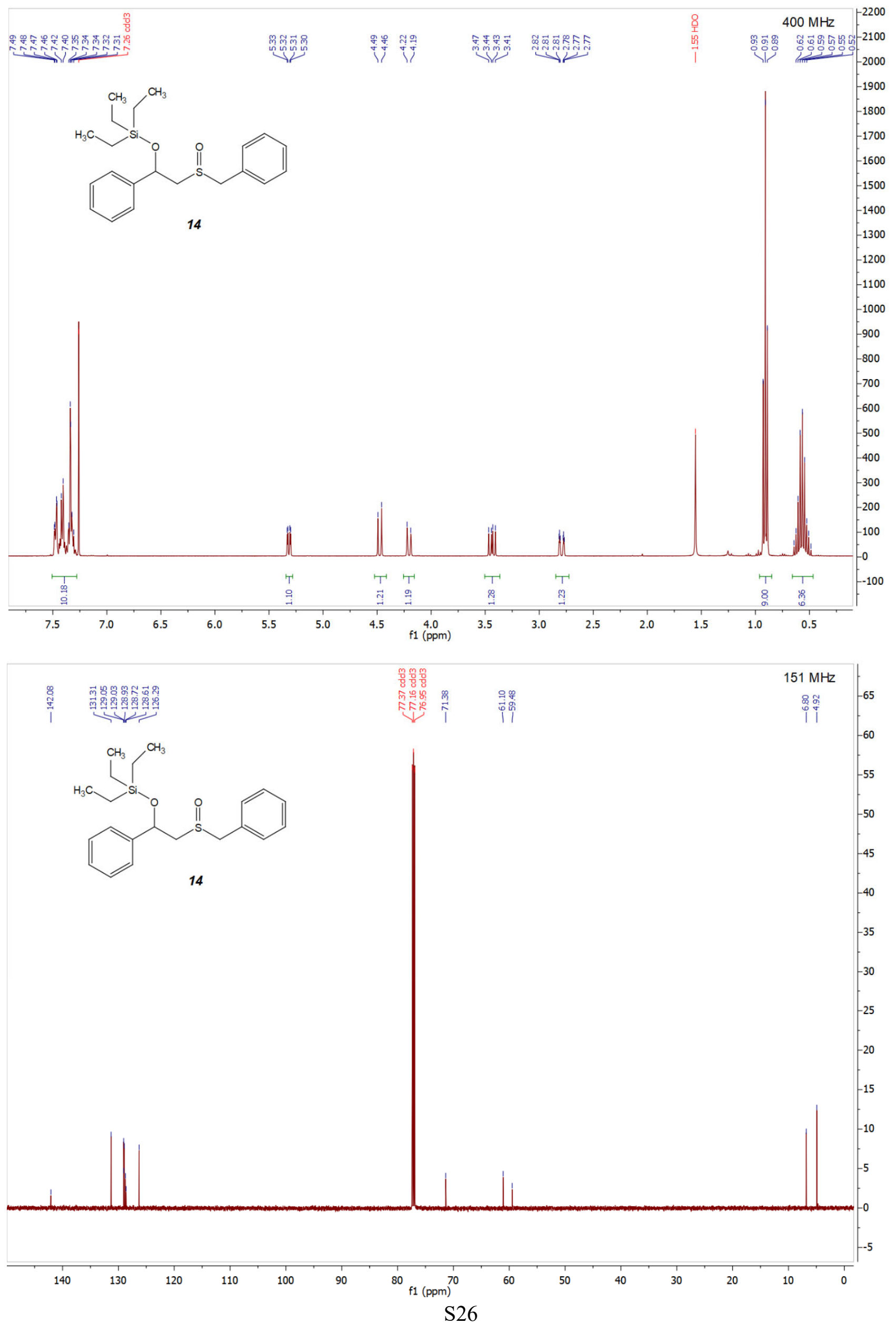




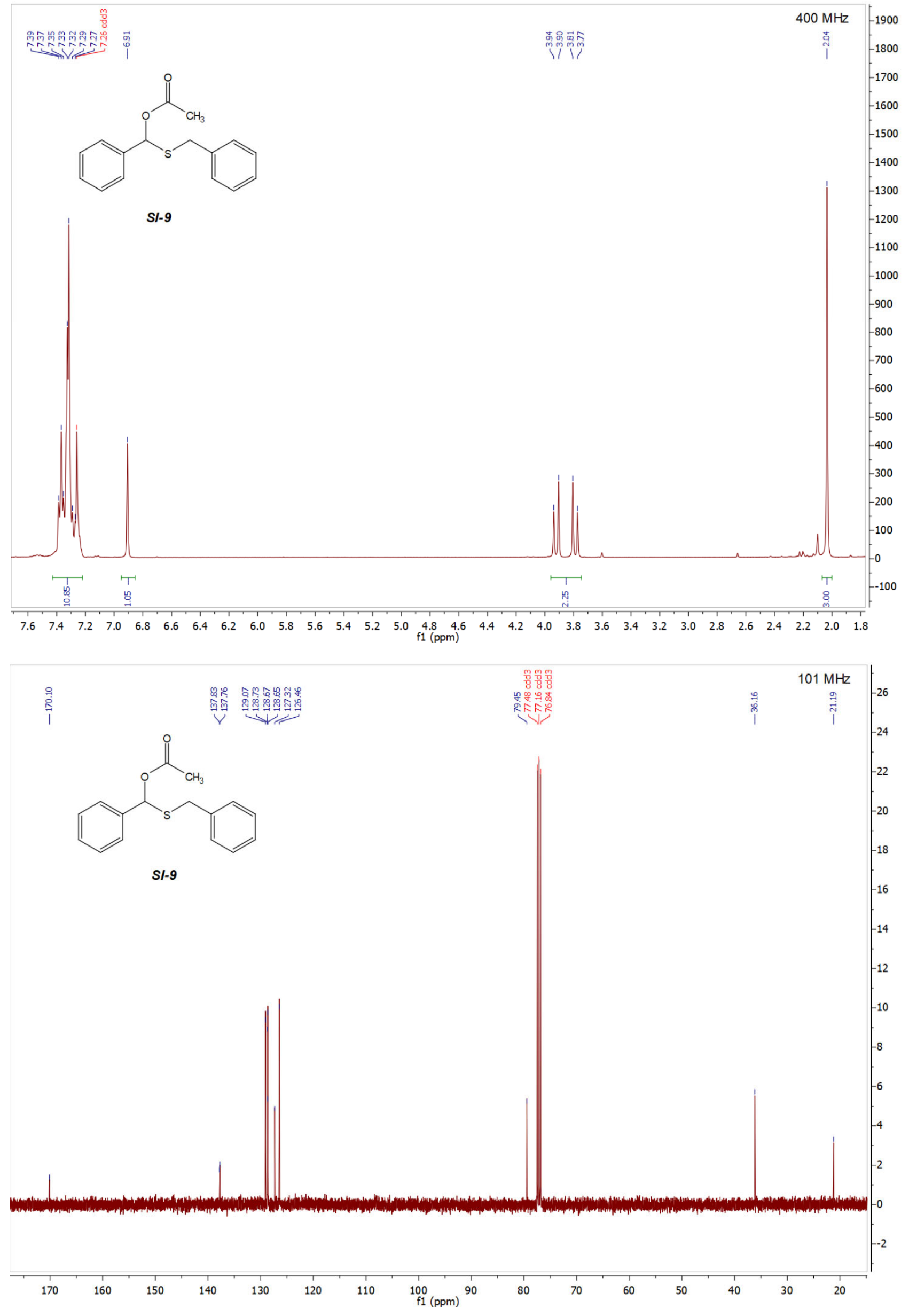




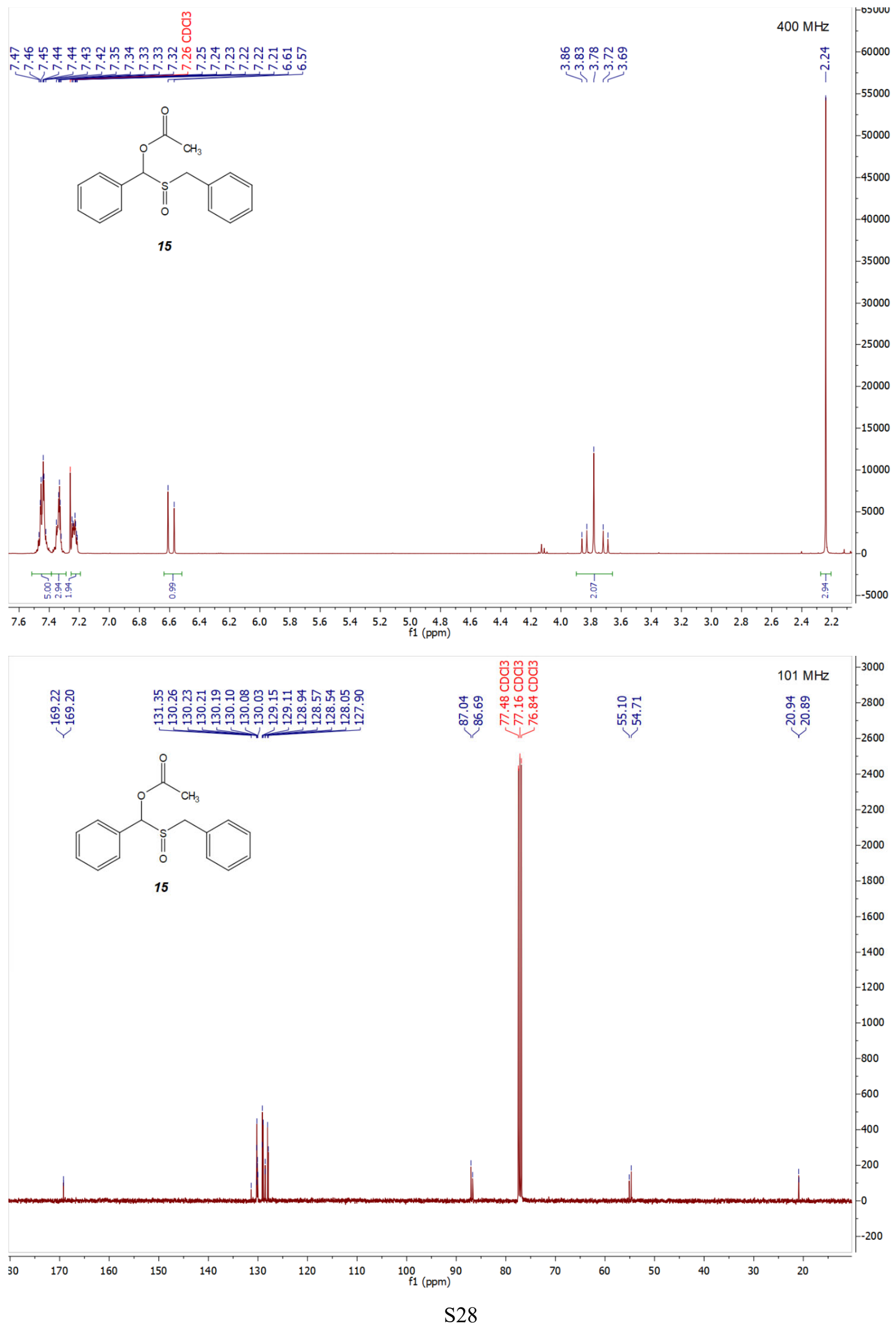




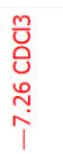
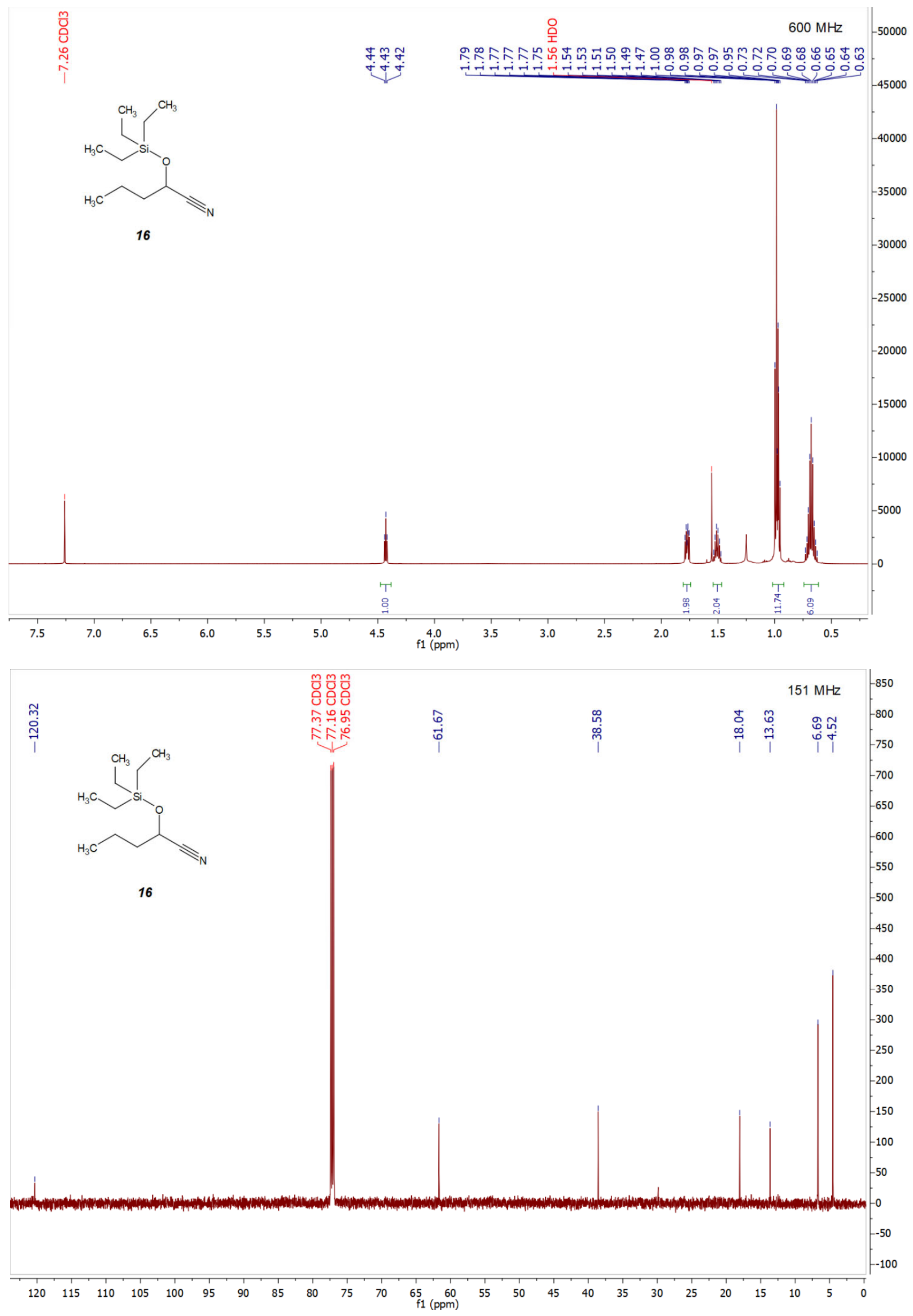


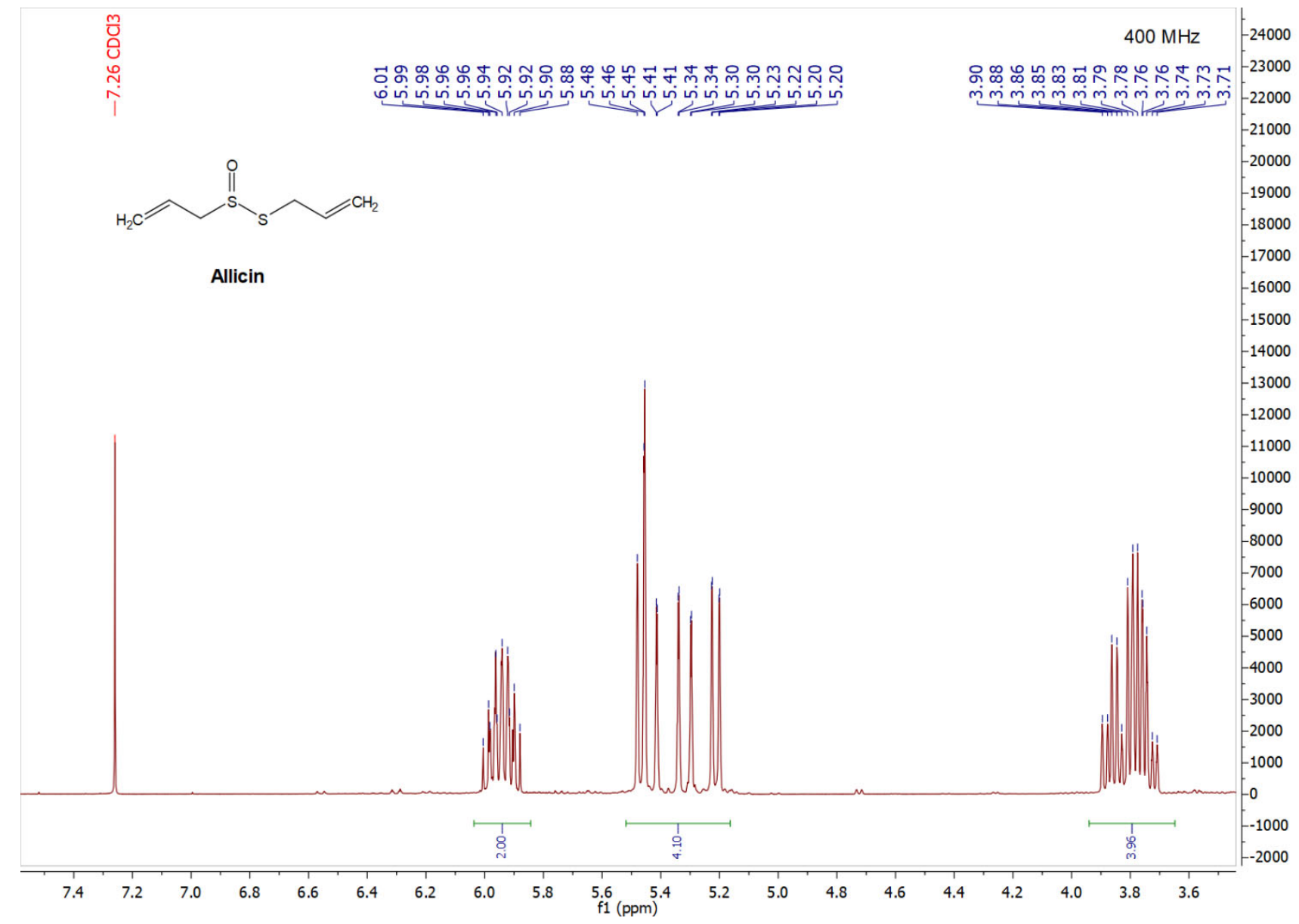

